\title{
B1.4
}

\section{Microwave and terahertz spectroscopy}

\author{
Geoffrey A Blake
}

\section{B1.4.1 Introduction}

Spectroscopy, or the study of the interaction of light with matter, has become one of the major tools of the natural and physical sciences during this century. As the wavelength of the radiation is varied across the electromagnetic spectrum, characteristic properties of atoms, molecules, liquids and solids are probed. In the optical and ultraviolet regions $(\lambda \sim 1 \mu \mathrm{m}$ up to $100 \mathrm{~nm}$ ) it is the electronic structure of the material that is investigated, while at infrared wavelengths $(\sim 1-30 \mu \mathrm{m})$ the vibrational degrees of freedom dominate.

Microwave spectroscopy began in 1934 with the observation of the $\sim 20 \mathrm{GHz}$ absorption spectrum of ammonia by Cleeton and Williams. Here we will consider the microwave region of the electromagnetic spectrum to cover the 1 to $100 \times 10^{9} \mathrm{~Hz}$, or 1 to $100 \mathrm{GHz}(\lambda \sim 30 \mathrm{~cm}$ down to $3 \mathrm{~mm})$, range. While the ammonia microwave spectrum probes the inversion motion of this unique pyramidal molecule, more typically microwave spectroscopy is associated with the pure rotational motion of gas phase species.

The section of the electromagnetic spectrum extending roughly from 0.1 to $10 \times 10^{12} \mathrm{~Hz}(0.1-10 \mathrm{THz}$, $3-300 \mathrm{~cm}^{-1}$ ) is commonly known as the far-infrared (FIR), submillimetre or terahertz (THz) region, and therefore lies between the microwave and infrared windows. Accordingly, $\mathrm{THz}$ spectroscopy shares both scientific and technological characteristics with its longer- and shorter-wavelength neighbours. While rich in scientific information, the FIR or THz region of the spectrum has, until recently, been notoriously lacking in good radiation sources - earning the dubious nickname 'the gap in the electromagnetic spectrum'. At its high-frequency boundary, most coherent photonic devices (e.g. diode lasers) cease to radiate due to the long lifetimes associated with spontaneous emission at these wavelengths, while at its low-frequency boundary parasitic losses reduce the oscillatory output from most electronic devices to insignificant levels. As a result, existing coherent sources suffer from a number of limitations. This situation is unfortunate since many scientific disciplines-including chemical physics, astrophysics, cosmochemistry and planetary/atmospheric science to name but a few-rely on high-resolution $\mathrm{THz}$ spectroscopy (both in a spectral and temporal sense). In addition, technological applications such as ultrafast signal processing and massive data transmission would derive tremendous enhancements in rate and volume throughput from frequency-agile $\mathrm{THz}$ synthesizers.

In general, THz frequencies are suitable for probing low-energy light-matter interactions, such as rotational transitions in molecules, phonons in solids, plasma dynamics, electronic fine structure in atoms, thermal imaging of cold sources and vibrational-rotation-tunnelling behaviour in weakly bound clusters. Within the laboratory, THz spectroscopy of a variety of molecules, clusters and condensed phases provides results that are critical to a proper interpretation of the data acquired on natural sources, and also leads to a better understanding of important materials - particularly hydrogen-bonded liquids, solids and polymers that participate in a variety of essential (bio)chemical processes.

For remote sensing, spectroscopy at $\mathrm{THz}$ frequencies holds the key to our ability to remotely sense environments as diverse as primaeval galaxies, star and planet-forming molecular cloud cores, comets and 
planetary atmospheres. In the dense interstellar medium characteristic of sites of star formation, for example, scattering of visible/UV light by sub-micron-sized dust grains makes molecular clouds optically opaque and lowers their internal temperature to only a few tens of Kelvin. The thermal radiation from such objects therefore peaks in the FIR and only becomes optically thin at even longer wavelengths. Rotational motions of small molecules and rovibrational transitions of larger species and clusters thus provide, in many cases, the only or the most powerful probes of the dense, cold gas and dust of the interstellar medium.

Since the major drivers of $\mathrm{THz}$ technology have been scientists, particularly physicists and astrophysicists seeking to carry out fundamental research, and not commercial interests, a strong coupling of technology development efforts for remote sensing with laboratory studies has long characterized spectroscopy at microwave and $\mathrm{THz}$ frequencies. In many respects the field is still in its infancy, and so this chapter will present both an overview of the fundamentals of microwave and $\mathrm{THz}$ spectroscopy as well as an assessment of the current technological state of the art and the potential for the future. We will begin with a brief overview of the general characteristics of $\mathrm{THz}$ spectrometers and the role of incoherent sources and detection strategies in the $\mathrm{THz}$ region, before turning to a more detailed description of the various coherent $\mathrm{THz}$ sources developed over the past decade and their applications to both remote sensing and laboratory studies.

\section{B1.4.2 Incoherent $\mathrm{THz}$ sources and broadband spectroscopy}

\section{B1.4.2.1 Principles and instrumentation}

Like most other fields of spectroscopy, research at THz frequencies in the first half of the twentieth century was carried out with either dispersive (i.e. grating-based) or Fourier transform spectrometers. The much higher throughput of Fourier transform spectrometers compared to those based on diffraction gratings has made THz Fourier transform spectroscopy, or THz FTS, the most popular incoherent technique for acquiring data over large regions of the $\mathrm{THz}$ spectrum. This is especially true for molecular line work where $\mathrm{THz}$ FTS resolutions of order $50-100 \mathrm{MHz}$ or better have been obtained [1]. With large-format detector arrays, such as those available at optical through near- to mid-infrared wavelengths, grating- or Fabry-Perot-based instruments can provide superior sensitivity [2], but have not yet been widely utilized at $\mathrm{THz}$ frequencies due to the great difficulty of fabricating arrays of $\mathrm{THz}$ detectors.

The components of $\mathrm{THz}$ spectrometers can be grouped into three main categories: sources (e.g. lasers, Gunn oscillators, mercury-discharge lamps), propagating components (e.g. lenses, sample cells, filters) and detectors (e.g. bolometers, pyroelectric detectors, photoacoustic cells). Propagating components in the FIR are well established (see [3] for an excellent overview of technical information). In the area of detectors, recent progress has placed them ahead of source technology. For example, spider-web Si bolometers developed by Bock et al [4] have an electrical NEP (noise-equivalent power) of $4 \times 10^{-17} \mathrm{~W} \mathrm{~Hz}^{-1 / 2}$ when cooled to $300 \mathrm{mK}$. For those who desire less exotic cryogenic options, commercially available Si-composite bolometers offer an electrical NEP of $1 \times 10^{-13} \mathrm{~W} \mathrm{~Hz}^{-1 / 2}$, operating at liquid helium temperature $(4.2 \mathrm{~K})$, and an electrical $\mathrm{NEP}$ of $3 \times 10^{-15} \mathrm{~W} \mathrm{~Hz}^{-1 / 2}$, operating at $1.2 \mathrm{~K}$ (pumped $\mathrm{L}_{\mathrm{He}}$ ). Combining these into large-format arrays remains a considerable technological challenge, although arrays of several tens of pixels on a side are now beginning to make their way into various telescopes such as the Caltech Submillimeter Observatory and the James Clerk Maxwell Telescope [5,6]. In addition, their electrical bandwidths are typically only between a few hundred hertz and $1 \mathrm{kHz}$. Fast-modulation schemes cannot therefore be used, and careful attention must be paid to $1 / f$ noise in experiments with Si bolometers. Hot-electron bolometers based on InSb offer electrical bandwidths of $\lesssim 1 \mathrm{MHz}$, but without cyclotron-assisted resonance, InSb THz bolometers cannot be used above $v=25-30 \mathrm{~cm}^{-1}$ [7]. Similarly, photoconductors based on Ga:Ge offer high electrical speed and good quantum efficiency, but due to the bandgap of the material are unusable below $50-60 \mathrm{~cm}^{-1}$ [8].

In practice, the NEP of a room-temperature $\mathrm{THz}$ spectrometer is usually limited by fluctuations (shotnoise) in the ambient blackbody radiation. Using an optical bandwidth $\Delta v=3 \mathrm{THz}$ (limited by, for example, 

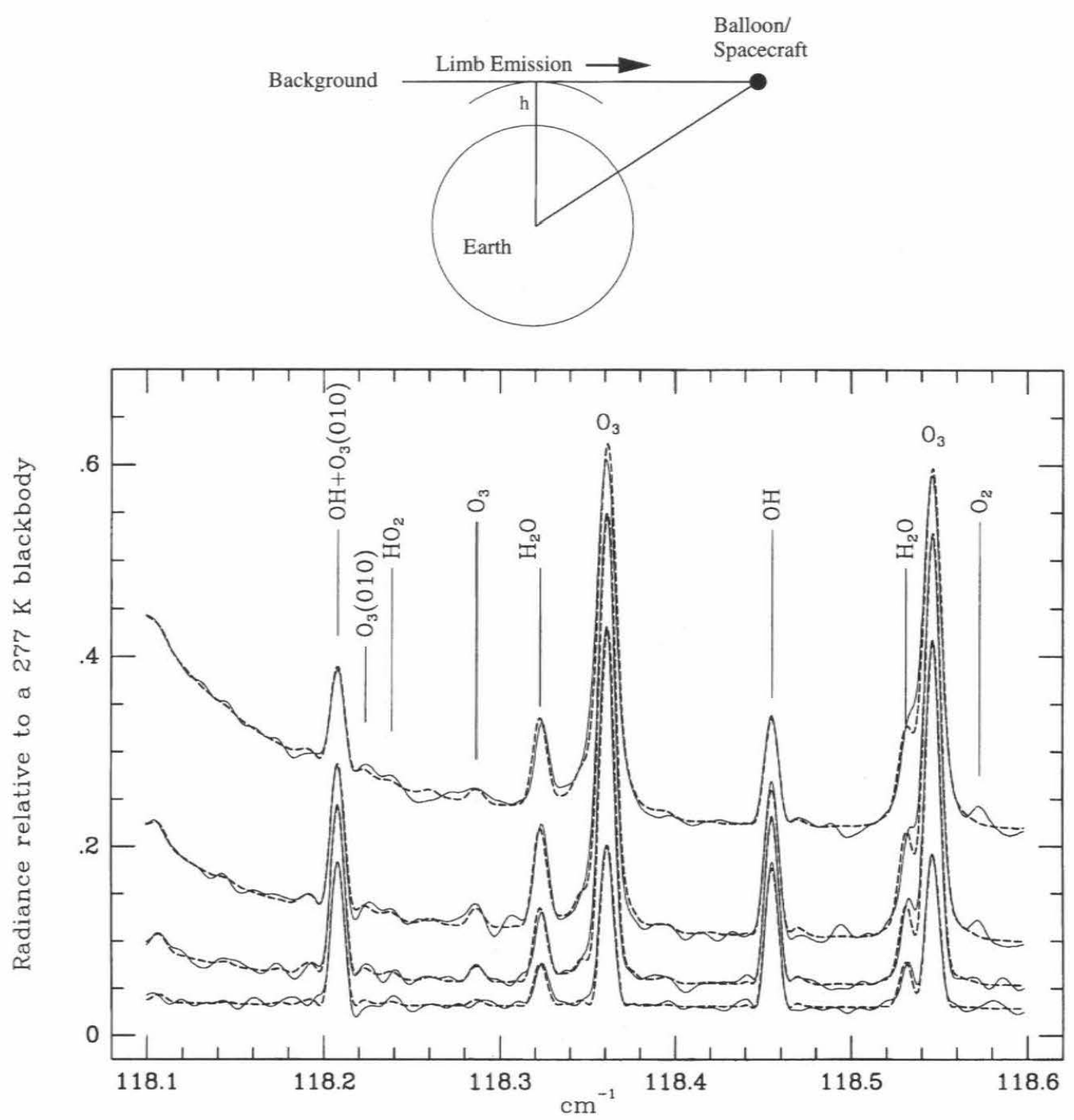

Figure B1.4.1. Top: schematic illustration of the observing geometry used for limb sounding of the Earth's atmosphere. Bottom: illustrative stratospheric $\mathrm{OH}$ emission spectra acquired by the SAO FIRS-2 far-infrared balloon-borne FTS in autumn 1989. The spectra are from a range of tangent heights ( $h=$ tangent height in the drawing), increasing toward the bottom, where the data are represented by solid curves; nonlinear least-square fits to the measurements, based on a combination of laboratory data, the physical structure of the stratosphere and a detailed radiative transfer calculation, are included as dashed curves. The $\mathrm{OH}$ lines are $\mathrm{F}_{1}\left({ }^{2} \Pi_{3 / 2}\right), 7 / 2^{-} \rightarrow 5 / 2^{+}$and $7 / 2^{+} \rightarrow 5 / 2^{-}$(the hyperfine structure is unresolved in these measurements). Other major contributing lines are also identified [10].

a polyethylene/diamond dust window), a field of view (at normal incidence) $\theta=9^{\circ}$ and a detecting diameter (using a so-called Winston cone, which condenses the incident radiation onto the detecting element) $d=$ $1.1 \mathrm{~cm}$, values that are typical for many laboratory applications, the background-limited NEP of a bolometer 
is given by

$$
\mathrm{NEP}=\frac{2 k T \pi d \sin (\theta / 2)(\Delta f \Delta \nu)^{1 / 2}}{\lambda}=0.2 \mathrm{pW}
$$

where $k$ is the Boltzmann constant, $T=300 \mathrm{~K}, \Delta f$ (the electronic amplified bandwidth) $=1 \mathrm{~Hz}$, and $\lambda$ (the band-centre wavelength) $\approx 200 \mu \mathrm{m}$. The equation above uses the Rayleigh-Jeans law, which is valid for $v \leq k T_{300} \mathrm{~K} \mathrm{~h}^{-1}=17 \mathrm{THz}$. Therefore, for laboratory absorption experiments, a typical FIR detector provides an estimated detection limit (NEP/source power) of $10^{-4}$ with a source output of $20 \mathrm{nW}$. In general, highsensitivity bolometers saturate at an incident-power level of $\approx 1 \mu \mathrm{W}$ or less, resulting in an ultimate detection limit of $10^{-7}$. For yet higher dynamic range, a filter element (e.g. cold grating, prism, or etalon) must be placed before the detector to reduce background noise, or the background temperature must be lowered. Note the $(\Delta v)^{1 / 2}$ dependence in equation B1.4.1, which means that the optical bandwidth must be reduced to $\sim 30 \mathrm{GHz}$ to drop the NEP by a factor of ten. Thus, unlike shorter wavelength regions of the electromagnetic spectrum, due to the high background luminosity in the $\mathrm{TH}$, spectroscopic sensitivity in the laboratory is limited by the source power, in comparison to the background power, incident on the detector-not by shot-noise of the available spectroscopic light sources. As higher-power light sources are developed at $\mathrm{THz}$ frequencies, lower-NEP detectors can be utilized that are less prone to saturation, and shot-noise will become the limiting factor, as it is in other regions of the electromagnetic spectrum.

For broadband THz FTS instruments this large background actually leads to a 'multiplex disadvantage' in that the room-temperature laboratory background can easily saturate the sensitive $\mathrm{THz}$ detectors that are needed to detect the feeble output of incoherent $\mathrm{THz}$ blackbody sources, which drop rapidly as the wavelength increases. The resulting sensitivity is such that signal-to-noise ratios in excess of 100 are difficult to generate at the highest feasible resolutions of $50-100 \mathrm{MHz}$ [1], which is still quite large compared to the 1-2 MHz Doppler-limited line widths at low pressure. For low-resolution work on condensed phases, or for the acquisition of survey spectra, however, THz FTS remains a popular technique. Beyond wavelengths of $\sim 1 \mathrm{~mm}$, the sensitivity of FTS is so low that the technique is no longer competitive with the coherent approaches described below.

\section{B1.4.2.2 THz FTS studies of planetary atmospheres}

Rather different circumstances are encountered when considering $\mathrm{THz}$ remote sensing of extraterrestrial sources. The major source of THz opacity in the Earth's atmosphere is water vapour, and from either high, dry mountain sites or from space there are windows in which the background becomes very small. Incoherent instruments which detect the faint emission from astronomical sources can therefore be considerably more sensitive than their laboratory counterparts. Again, grating- or etalon-based and FTS implementations can be considered, with the former being preferred if somewhat coarse spectral resolution is desired or if large-format detector arrays are available.

In planetary atmospheres, a distinct advantage of $\mathrm{THz}$ studies over those at optical and infrared wavelengths is the ability to carry out spectroscopy without a background or input source such as the sun. Global maps of a wide variety of species can therefore be obtained at any time of day or night and, when taken at high enough spectral resolution, the shapes of the spectral lines themselves also contain additional information about vertical abundance variations and can be used to estimate the atmospheric temperature profile. The 'limb sounding' geometry, in which microwave and THz emission from the limb of a planetary atmosphere is imaged by an orbiting spacecraft or a balloon, is particularly powerful in this regard, and excellent reviews are available on this subject [9]. The observing geometry is illustrated in figure B1.4.1, which also presents a portion of the Earth's stratospheric emission spectrum near $118 \mathrm{~cm}^{-1}$ obtained by the balloon-borne Smithsonian Astrophysical Observatory limb-sounding THz FTS [10]. 


\section{B1.4.3 Coherent THz sources and heterodyne spectroscopy}

\section{B1.4.3.1 Principles and instrumentation}

The narrow cores of atmospheric transitions shown in figure B1.4.1 can be used, among other things, to trace the wind patterns of the upper atmosphere. For such work, or for astronomical remote-sensing efforts, resolutions of the order of $30-300 \mathrm{~m} \mathrm{~s}^{-1}$ are needed to obtain pressure broadening or kinematic information, which correspond to spectral resolutions of $(v / \Delta v) \sim 1-10 \times 10^{6}$. Neither FTS nor grating-based spectrometers can provide resolution at this level and so other techniques based on coherent radiation sources must be used. The most important of these is called heterodyne spectroscopy. Heterodyne spectroscopy uses nonlinear detectors called mixers, in order to downconvert the high-frequency $\mathrm{THz}$ radiation into radiofrequency or microwave signals that can be processed using commercial instrumentation. An outline of a heterodyne receiver is presented in figure B1.4.2. In such a receiver, an antenna (dish) collects radiation from space, and this radiation is focused onto a detector operating in heterodyne mode, which means that the incoming signal is mixed with the output of a coherent source (called the local oscillator, or LO). Now, if a device can be constructed that responds quadratically, rather than linearly, to the two input beams, the output, $S(t)$, of such a device is given by

$$
\begin{aligned}
S(t) \propto & e(t)^{2} \\
S(t) \propto & 1 / 2\left(E_{1}^{2}+E_{1}^{2}\right)+1 / 2\left[E_{1}^{2} \cos \left(2 \omega_{1} t\right)+E_{2}^{2} \cos \left(2 \omega_{2} t\right)\right] \\
& +E_{1} E_{2} \cos \left[\left(\omega_{1} t+\omega_{2} t\right)+\left(\phi_{1}+\phi_{2}\right)\right] \\
& +\underbrace{E_{1} E_{2} \cos \left[\left(\omega_{1}-\omega_{2}\right) t+\left(\phi_{1}-\phi_{2}\right)\right]}_{\text {DIFFERENCE-FREQUENCY FIELD }}
\end{aligned}
$$

where the identities $2 \cos \alpha \cos \beta=\cos (\alpha+\beta)+\cos (\alpha-\beta)$ and $\cos ^{2} \alpha=1 / 2(1+\cos 2 \alpha)$ are invoked. The last term is a field oscillating at a frequency equal to the difference between the two incidental fields - representing the beat-note between $v_{1}$ and $v_{2}$.

If $v_{1}=v_{\mathrm{LO}}$ and $v_{2}=v_{\mathrm{SKY}} \approx v_{\mathrm{LO}}$, the sum frequency lies at THz frequencies, while the difference lies at radio or microwave frequencies, and is called the intermediate frequency, or IF. The IF can be amplified and recorded (for example, on a spectrum analyser or by a digital correlator or filterbank) across a range of frequencies simultaneously. The fact that the IF power is proportional to the product of the remote-signal power and the LO power results in two main advantages: (a) the signal-to-noise ratio is enhanced by using an LO power that is much higher than that of the remote signal and (b) the spectral resolution is set by the linewidth of the LO, which can be as narrow as desired.

Among the first $\mathrm{THz}$ mixers to be constructed were those based on room-temperature Schottky diodes [11]. Over the past decade, new mixers based on superconducting tunnel junctions have been developed that have effective noise levels only a few times the quantum limit of $T_{\text {mixer }}=h v / k$ [12]. However, certain conditions must be met in order to exploit these advantages. For example, while the LO power should be strong compared to the remote signal and to the noise, it must not be so strong as to saturate the detecting element. Roughly speaking, the optical coupled LO power level is about $1 \mu \mathrm{W}$ for SIS (superconductorinsulator-superconductor) mixers and $100 \mathrm{nW}$ for superconductive Nb HEBs (hot-electron bolometers, or transition edge bolometers, TEBs). In addition, since the overlap between the remote signal and the LO is important, the spatial distribution of the LO output must be well coupled to the receiver's antenna mode. Although this requirement imposes experimental complexity, it also provides excellent rejection of ambient background radiation.

As noted above, at $\mathrm{THz}$ frequencies the Rayleigh-Jeans approximation is a good one, and it is typical to report line intensities and detector sensitivities in terms of the Rayleigh-Jeans equivalent temperatures. In frequencies range where the atmospheric transmission is good, or from airborne or space-borne platforms, 


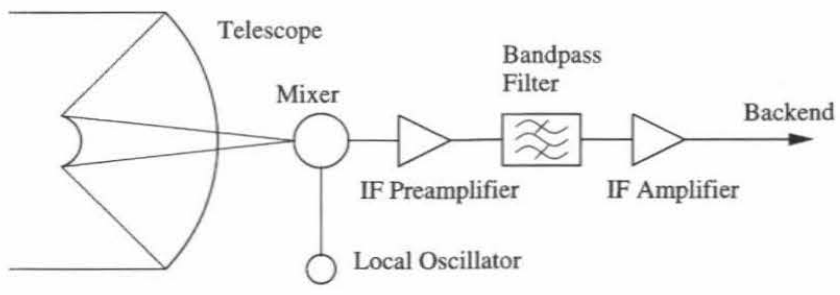

(A)

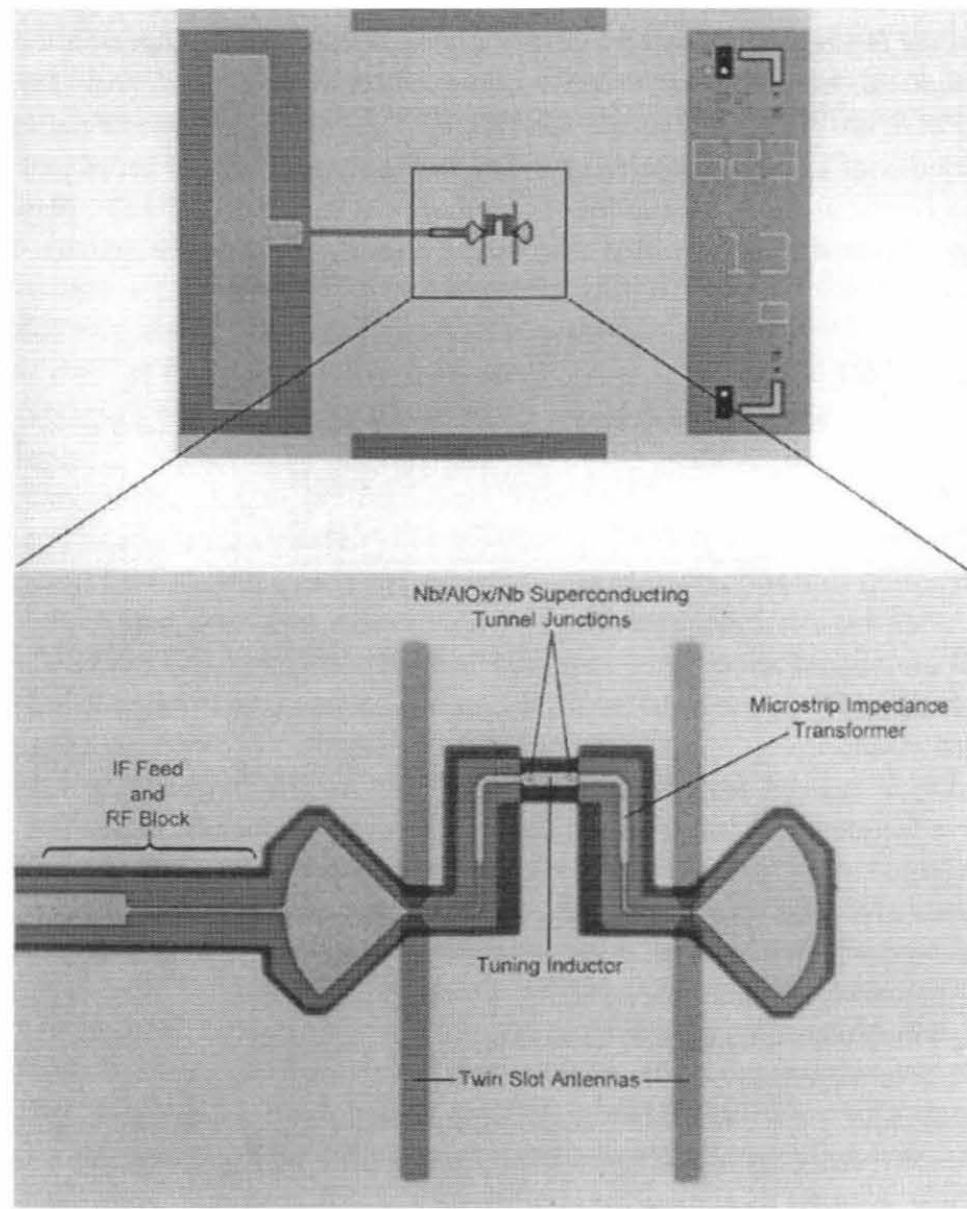

(B)

Figure B1.4.2. (A) Basic components of an astronomical heterodyne receiver. The photomicrograph in (B) presents the heart of a quasi-optical SIS mixer and its associated superconducting tuning circuits, while the image in (C) shows the fully assembled mixer, as it would be incorporated into a low-temperature cryostat (J Zmuidzinas, private communication).

the effective background temperature is only a few tens of Kelvin. Under such conditions, SIS mixers based on $\mathrm{Nb}$, a particular implementation of which is pictured in figure B1.4.2, can now perform up to $1.0 \mathrm{THz}$ with $T_{n \text {,mixer }}=130 \mathrm{~K}$ [13]. The earliest SIS microwave and millimetre-wave receivers utilized waveguide 
a)

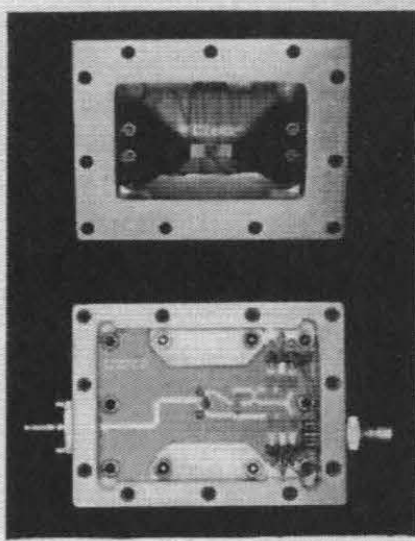

c)

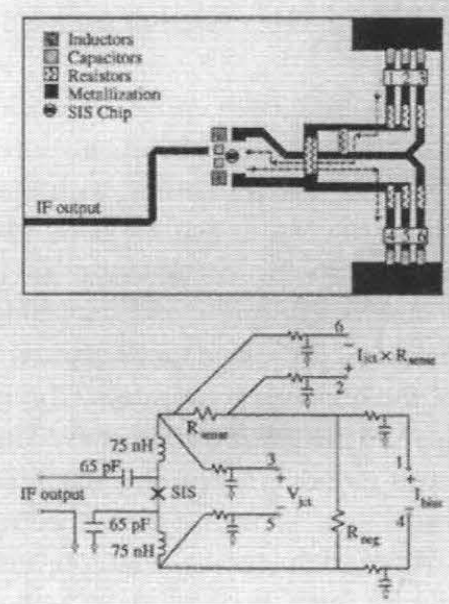

b)
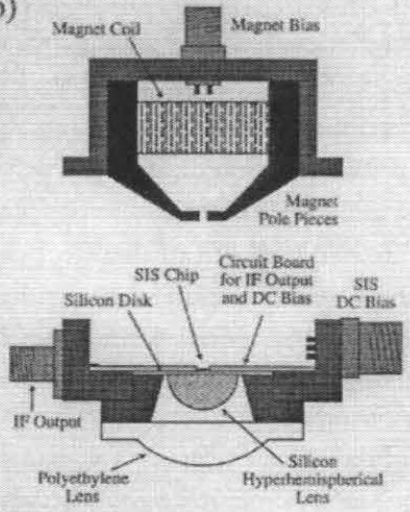

d)

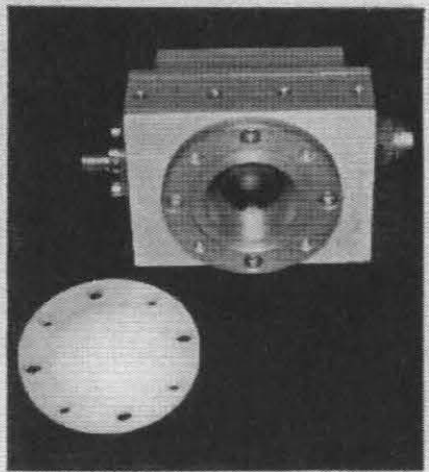

(C)

Figure B1.4.2. (Continued)

components, but as the operating frequencies have been pushed into the $\mathrm{THz}$ region, quasi-optical designs such as those shown in figure B1.4.2 become attractive. Such designs may also be easier to incorporate into $\mathrm{THz}$ receiver arrays. Recently, alternative superconducting $\mathrm{Nb}$ hot-electron mixers that rely on a diffusionbased relaxation mechanism have been demonstrated with $T_{\text {mixer }}=750 \mathrm{~K}$ between 1 and $2.5 \mathrm{THz}$ [14]. These devices are expected to operate up to at least several tens of $\mathrm{THz}$-if coherent sources are available as LOs. Thus, THz source technology plays a key role in setting the spectroscopic sensitivity for both laboratory and remote-sensing experiments.

\section{B1.4.3.2 THz remote sensing with heterodyne receivers}

Heterodyne spectroscopy has been particularly critical to the study of the Earth's stratosphere, where the improved resolution and sensitivity compared to the FTS spectra shown in figure B1.4.1 have led to the collection of global maps of species important to ozone chemistry and atmospheric dynamics $\left(\mathrm{O}_{3}, \mathrm{ClO}, \mathrm{SO}_{2}\right.$, 
$\mathrm{H}_{2} \mathrm{O}, \mathrm{O}_{2}$, etc: see [9] for an extended overview), and of the dense interstellar medium. Although human beings have been systematically observing astronomical objects for thousands of years, until the advent of radioastronomy in the 1960s we possessed little knowledge of what, if anything, exists in the space between stars. Optical observations revealed only stars, galaxies and nebulae; if matter existed in the vast, dark interstellar medium, it was not detectable. However, the discovery of the first polyatomic microwave in the interstellar medium, water $\left(\mathrm{H}_{2} \mathrm{O}\right)$, ammonia $\left(\mathrm{NH}_{3}\right)$ and formaldehyde $\left(\mathrm{H}_{2} \mathrm{CO}\right)$, by microwave remote sensing (in 1968 [15]) set off an exciting era of discovery.

To date, researchers have identified more than 100 different molecules, composed of up to 13 atoms, in the interstellar medium [16]. Most were initially detected at microwave and (sub)millimetre frequencies, and the discoveries have reached far beyond the mere existence of molecules. Newly discovered entities such as diffuse interstellar clouds, dense (or dark) molecular clouds and giant molecular cloud complexes were characterized for the first time. Indeed, radioastronomy (which includes observations ranging from radio to submillimetre frequencies) has dramatically changed our perception of the composition of the universe. Radioastronomy has shown that most of the mass in the interstellar medium is contained in so-called dense molecular clouds, which have tremendous sizes of 1-100 light years, average gas densities of $10^{2}-10^{3} \mathrm{~cm}^{-3}$, and temperatures in the range of $10-600 \mathrm{~K}$. An overview of the $\mathrm{THz}$ emission from a cold, dense interstellar cloud is presented in figure B1.4.3 [17].

In addition to striking differences from one cloud to another, each dense molecular cloud is inhomogeneous, containing clumps, or cores, of higher-density material situated within envelopes of somewhat lower density. Many of these higher-density cores are active sites of star formation, with the youngest stars being detectable only in the IR or FIR. Star formation is of major interest in astrophysics, and it contains a wealth of interesting chemical reactions and physical phenomena (for excellent reviews, see [18-20]). Optical observations are unable to characterize interstellar clouds due to absorption and scattering, both of which have an inverse wavelength dependence, by the pervasive dust particles inside these clouds. Thus, microwave and $\mathrm{THz}$ spectroscopy is responsible for identifying most of the hundred or so interstellar molecules to data, and continues to dominate the fields of molecular astrophysics and interstellar chemistry. The power of heterodyne spectroscopy in examining the differences between dense clouds undergoing star (and presumably planet) formation is shown in the right panel of figure $\mathrm{B} 1.4 .3$, which depicts the $345 \mathrm{GHz}$ spectral line surveys of three regions of the W3 giant molecular cloud complex [21]. From such studies, which reveal dramatic differences in the $\mathrm{THz}$ spectrum of various objects, molecular astrophysicists hope to classify the evolutionary state of the cloud, just as optical spectra are used to classify stars.

High angular resolution studies with modern $\mathrm{THz}$ telescopes and interferometric arrays can even probe the material destined to become part of planetary systems. In the accretion discs around young stars, for example, a range of simple organic species have now been detected at (sub)millimetre wavelengths [22]. Such accretion disks are the assembly zones of planets, and the first steps in $\mathrm{THz}$ imaging their outer regions and in understanding the means by which they evolve have been taken [23]. The physical and chemical conditions in these objects can now be compared to that observed in primitive solar system objects such as comets and icy satellites. The recent apparitions of comets Hyakutake and Hale-Bopp, for example, have provided a wealth of new observations at IR, THz and microwave frequencies that have led to a much improved understanding of the origin and evolution of planetesimals in the outer solar system [24]. Future work in high-resolution THz imaging will be dramatically enhanced by the Atacama Large Millimeter Array (ALMA), which will operate over the $1 \mathrm{~cm}$ to $350 \mu \mathrm{m}$ interval at an altitude of $16000 \mathrm{ft}$ in the Atacama desert of northern Chile [25].

Ultimately, studies from ground-based observatories are limited by absorption in the Earth's atmosphere. For example, no studies above $\approx 1 \mathrm{THz}$ are possible even from mountain-top observatories. Two major instruments, SOFIA and FIRST, are poised to change this situation dramatically. SOFIA (for Stratospheric Observatory For Infrared Astronomy [26]) will carry a $2.7 \mathrm{~m}$ telescope in a 747SP aircraft to altitudes of $41000-45000$ feet. At these altitudes, nearly $60-70 \%$ of the $\mathrm{THz}$ spectrum up to the mid-IR is accessible 


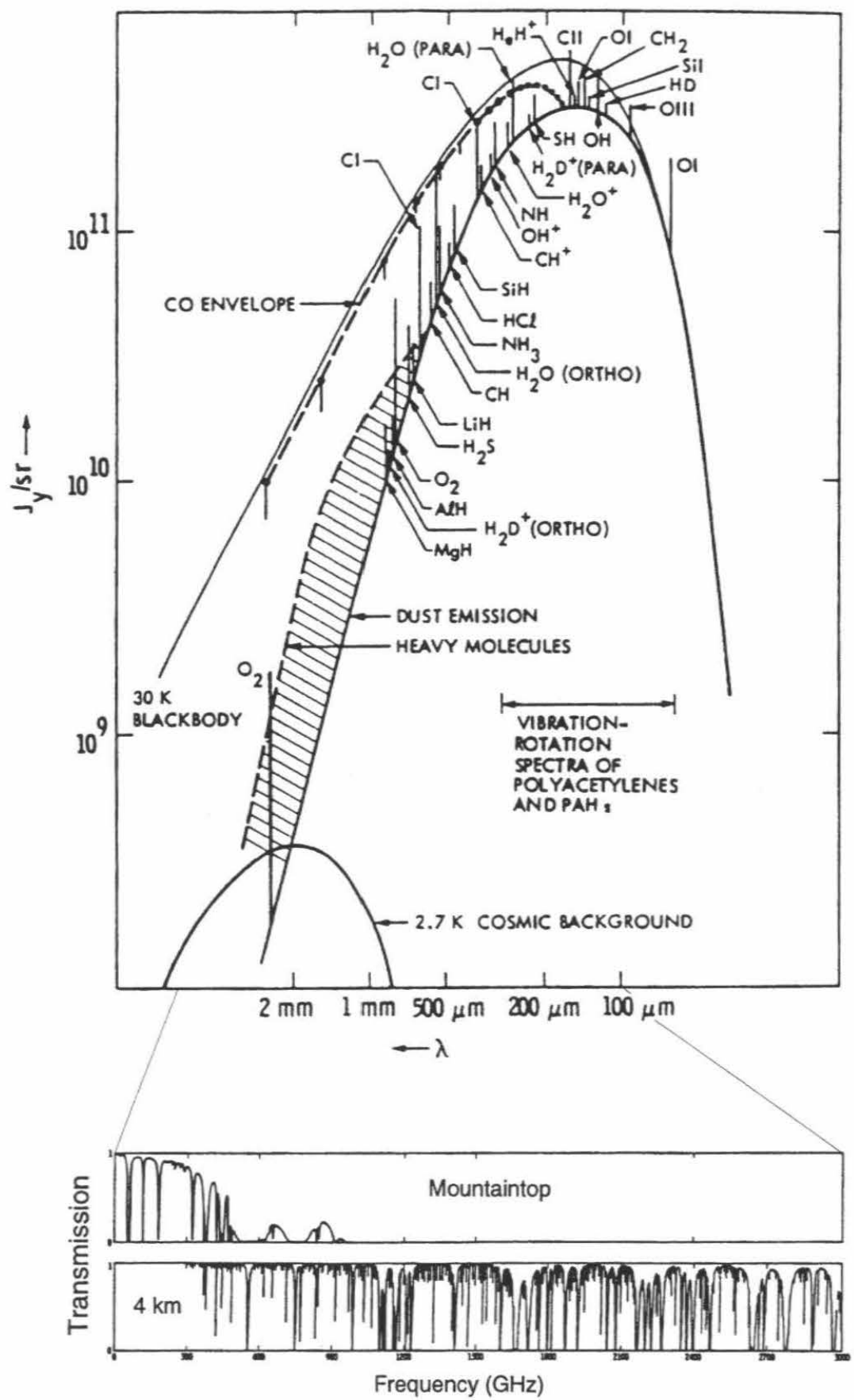

(a)

Figure B1.4.3. (a) A schematic illustration of the $\mathrm{THz}$ emission spectrum of a dense molecular cloud core at $30 \mathrm{~K}$ and the atmospheric transmission from ground and airborne altitudes (adapted, with permission, from [17]). (b) The results of $345 \mathrm{GHz}$ molecular line surveys of three cores in the W3 molecular cloud; the graphics at left depict the evolutionary state of the dense cores inferred from the molecular line data [21]. 


\section{CHEMICAL EVOLUTION IN THE W 3 MASSIVE STAR-FORMING REGION}
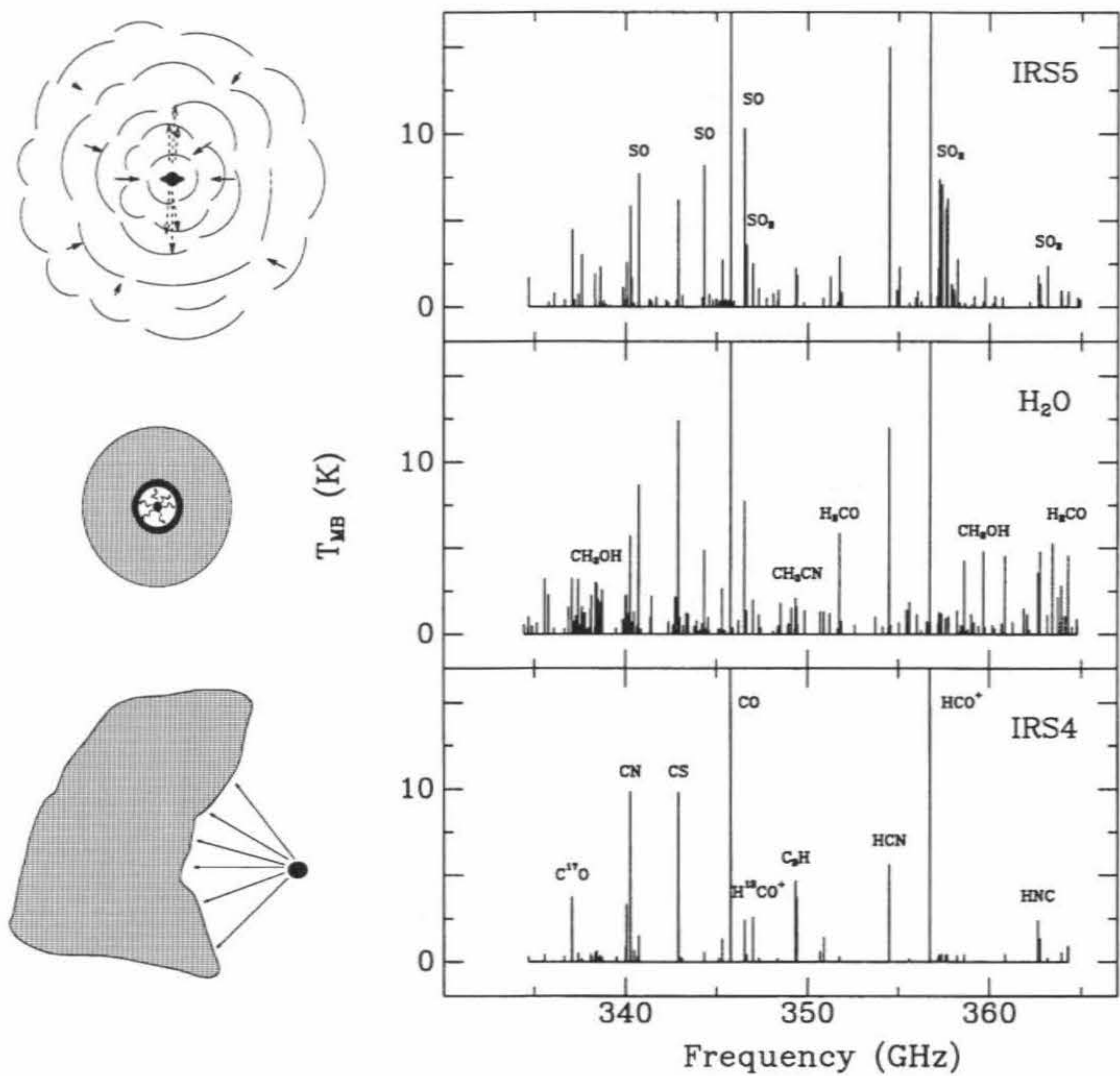

(b)

Figure B1.4.3. (Continued)

(figure B1.4.3), and both incoherent and heterodyne spectrometers are being constructed as part of the initial instrument suite. SOFIA will become operational in 2002. On somewhat longer timescales, FIRST (the FarInfraRed Space Telescope [27]) will carry $0.4-1.2 \mathrm{THz}$ SIS and 1.2-1.9/2.4-2.7 THz antenna coupled HEB receivers at the focal plane of a $4 \mathrm{~m}$ telescope into space. Like SOFIA, FIRST will also include incoherent spectrometers and imagers to take advantage of the low background flux and to survey larger regions of the $\mathrm{THz}$ spectrum and sky.

\section{B1.4.4 Spectroscopy with tunable microwave and THz sources}

\section{B1.4.4.1 Principles and background}

Long before the invention of the laser, coherent radiation sources such as electron beam tubes (e.g. klystrons and backward wave oscillators, or BWOs) were in use by microwave spectroscopists to examine the direct absorption rotational spectroscopy of molecules. Being the interface between microwave spectroscopy (generally associated with low-energy rotational transitions of molecules) and mid-IR spectroscopy (generally associated with vibrational transitions of molecules), $\mathrm{THz}$ spectra such as those outlined in figure B1.4.3 probe 
the high-frequency rotations of molecules and certain large-amplitude vibrational motions. As the rotational energy levels of a molecule depend largely on its moment of inertia, which is determined by the molecular structure, high-resolution spectroscopy of gas phase molecules provides the most precise information available on molecular geometries.

Rotational transition frequencies acquired in the $\mathrm{THz}$ region expand upon and complement those acquired in the microwave. Two types of molecules undergo rotational transitions that fall in the FIR: molecules with rotation about an axis having a small moment of inertia, and molecules in high- $J$ states. FIR spectra of the first type of molecules are important for determining their equilibrium geometry, as many light molecules $\left(\mathrm{H}_{2} \mathrm{O}, \mathrm{NH}_{3}, \mathrm{HF}\right.$, etc) only have transitions in the submillimetre and FIR regions. Due to the high rotational energy in the second type of species (high- $J$ molecules), interactions between the vibrational and rotational motions, namely, centrifugal distortion and Coriolis perturbations, become important. Given high enough spectral resolution and accuracy $\left(\Delta v / v \leq 10^{-5}\right)$, shifts in rotational frequencies and changes in selection rules resulting from these interactions become significant. Thus, FIR spectroscopy of high- $J$ transitions enables detailed characterization of molecular Hamiltonians far beyond the rigid rotor approximation, giving more accurate zero-point rotational constants and rough estimates of the shapes of potential energy surfaces. Finally, for very large molecules or weakly bound clusters, the softest vibrational degrees of freedom can be proved at $\mathrm{THz}$ frequencies, as is outlined in greater detail below.

\section{B1.4.4.2 Fourier transform microwave spectroscopy}

At microwave frequencies, direct absorption techniques become less sensitive than those in the $\mathrm{THz}$ region due to the steep dependence of the transition intensities with frequency. A variant of heterodyne spectroscopy, pioneered by Flygare and Balle [28], has proven to be much more sensitive. In this approach, molecules are seeded into or generated by a pulsed molecular beam which expands into a high- $Q$ microwave cavity. The adiabatic expansion cools the rotational and translation degrees of freedom to temperatures near 1-10 K, and thus greatly simplifies the rotational spectra of large molecules. In addition, the low-energy collisional environment of the jet can lead to the growth of clusters held together by weak intermolecular forces.

A microwave pulse from a tunable oscillator is injected into the cavity by an antenna, and creates a coherent superposition of rotational states. In the absence of collisions, this superposition emits a freeinduction decay signal, which is detected with an antenna-coupled microwave mixer similar to those used in molecular astrophysics. The data are collected in the time domain and Fourier transformed to yield the spectrum whose bandwidth is determined by the quality factor of the cavity. Hence, such instruments are called Fourier transform microwave (FTMW) spectrometers (or Flygare-Balle spectrometers, after the inventors). FTMW instruments are extraordinarily sensitive, and can be used to examine a wide range of stable molecules as well as highly transient or reactive species such as hydrogen-bonded or refractory clusters [29, 30].

An outline of an FTMW instrument used in the study of large, polar, carbonaceous species is shown in figure B1.4.4. In this instrument, the FTMW cavity is mated to a pulsed electric discharge/supersonic expansion nozzle [31,32]. Long-chain carbon species, up to that of $\mathrm{HC}_{17} \mathrm{~N}$, as shown in figure $\mathrm{B} 1.4 .4$, can be studied with this technique, as can a wide variety of other molecules and clusters. With the jet directed along the axis of the cavity, the resolution is highly sub-Doppler, with the slight complication that a Doppler doublet is formed by the difference between the laboratory and molecular beam reference frames. Studies of the rotational spectra of hydrogen-bonded clusters have also been carried out by several groups using FTMW instruments, a topic we shall return to later. FT-THz instruments can, in principle, be built using the highly sensitive THz SIS or HEB mixers outlined above, and would have extraordinary sensitivities. In order to saturate the rotational or rovibrational transitions, however, high-power $\mathrm{THz}$ oscillators are needed, but are not yet available. 


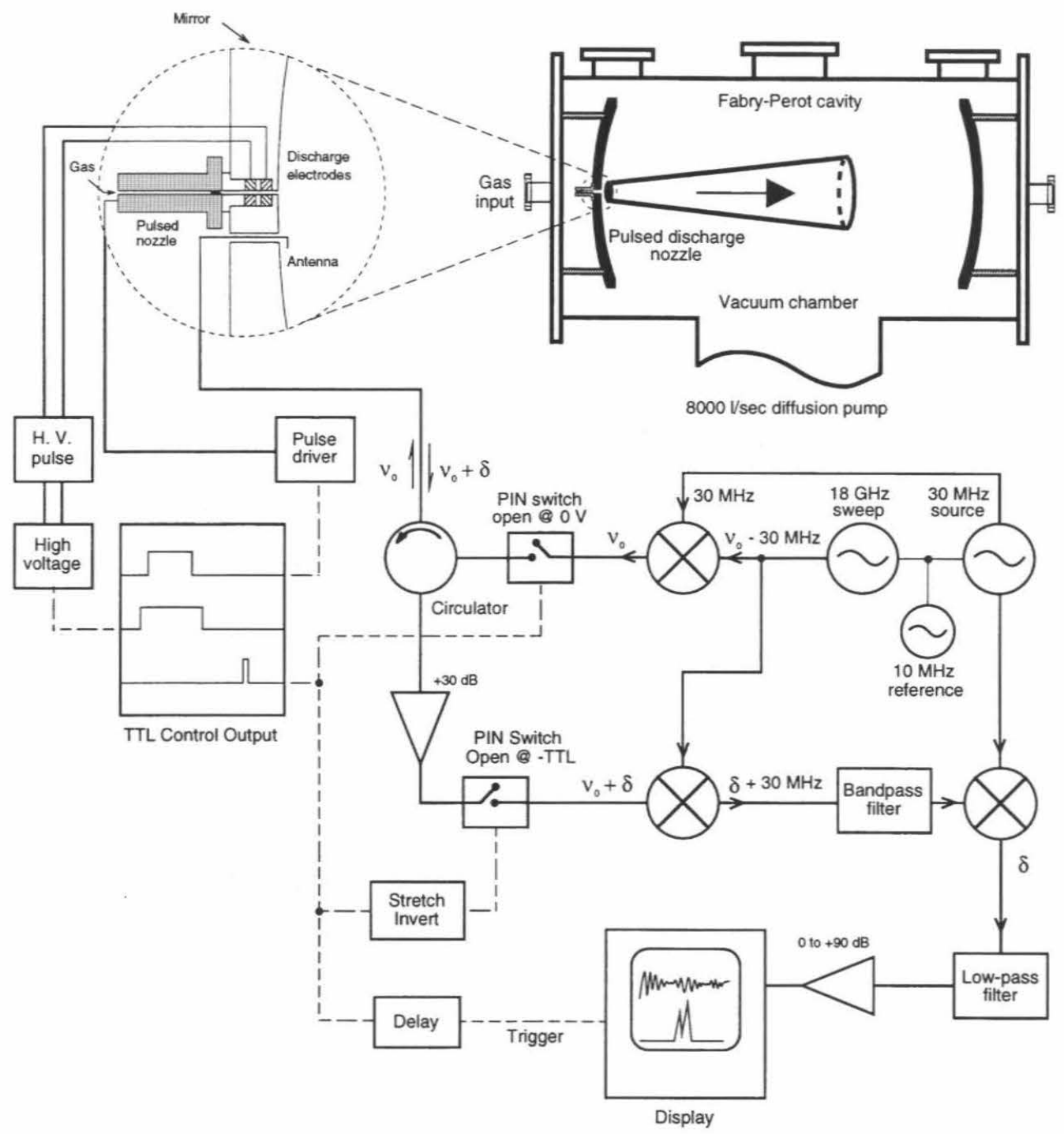

(a)

Figure B1.4.4. (a) An outline of the Harvard University electric discharge supersonic nozzle/Fourier transform microwave spectrometer. (b) The rotational states of $\mathrm{HC}_{17} \mathrm{~N}$ observed with this apparatus [31].

\section{B1.4.4.3 CW THz sources and molecular spectroscopy}

At frequencies up to $\sim 150-200 \mathrm{GHz}$, solid-state sources such as YIG-tuned oscillators or Gunn diode oscillators are now available with power outputs of up to $100 \mathrm{~mW}$. The harmonic generation of such millimetre-wave sources is relatively efficient for doubling and tripling $(\geq 10-15 \%)$, but for higher harmonics the power drops rapidly $\left(P_{\text {out }}(1 \mathrm{THz}) \leq 0.1-10 \mu \mathrm{W}\right)$. Nevertheless, harmonic generation was used as early as the $1950 \mathrm{~s}$ to record the submillimetre wave spectra of stable molecules [33]. Harmonics from optimized solid-state millimetre-wave sources are now used to drive astronomical heterodyne receivers up to 900-1100 GHz [34], and the prospects for operation up to $2-3 \mathrm{THz}$ are promising. 
$\mathrm{H}-\mathrm{C} \equiv \mathrm{C}-\mathrm{C} \equiv \mathrm{C}-\mathrm{C} \equiv \mathrm{C}-\mathrm{C} \equiv \mathrm{C}-\mathrm{C} \equiv \mathrm{C}-\mathrm{C} \equiv \mathrm{C}-\mathrm{C} \equiv \mathrm{C}-\mathrm{C} \equiv \mathrm{C}-\mathrm{C} \equiv \mathrm{N}$
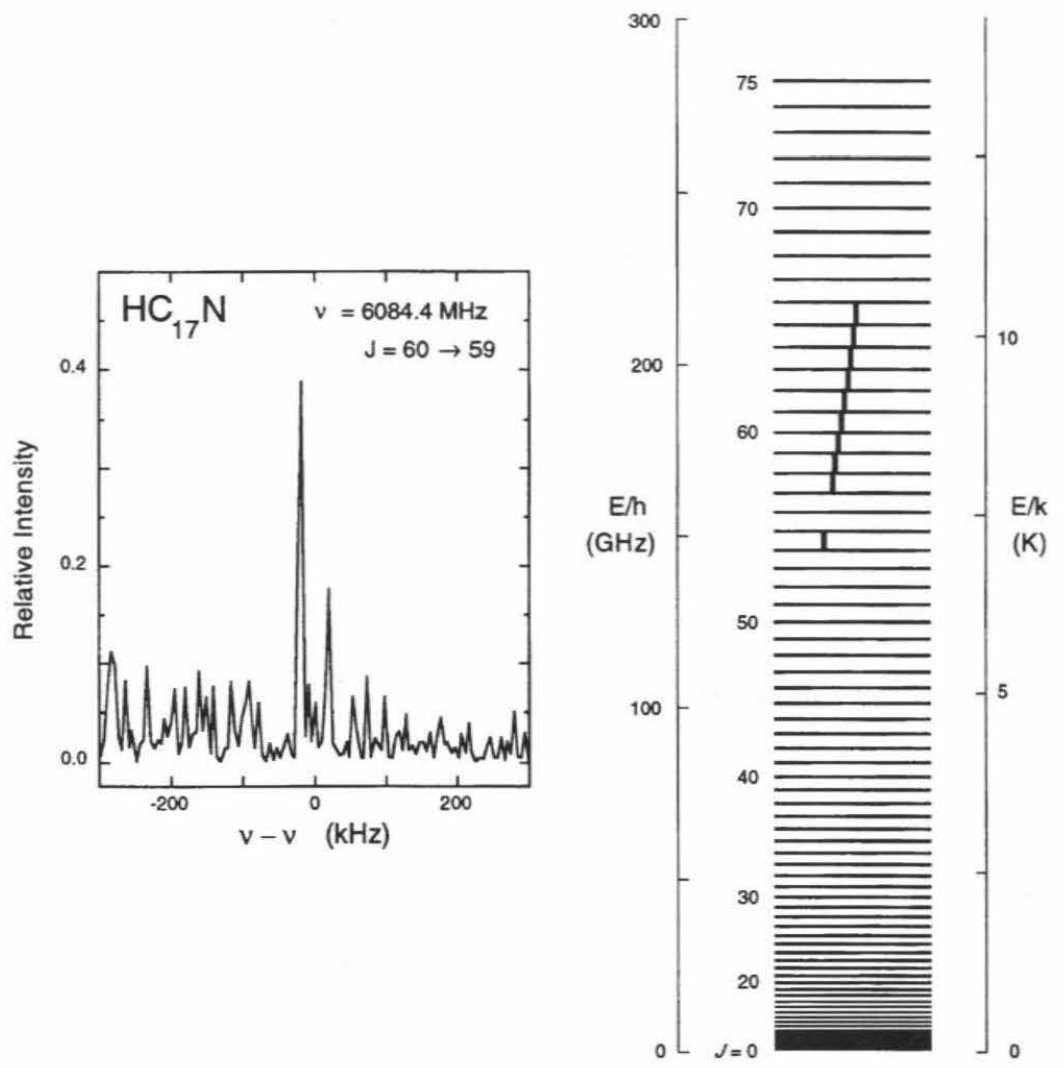

(b)

Figure B1.4.4. (Continued)

Even higher output power $(\sim 1-10 \mathrm{~mW})$ is available from rapidly tunable BWOs up to $1-15 \mathrm{THz}$. BWOs are capable laboratory sources where they operate, and offer wide tunability and excellent spectral purity, especially when phase locked to the harmonics of lower-frequency microwave or millimetre-wave oscillators [35]. The high output power of BWOs and the relatively strong intrinsic strengths of pure rotational transitions of polar molecules gives BWO spectrometers very high sensitivity, and also enables them to utilize nonlinear methods such as Lamb dip spectroscopy. An example for the CO molecule is presented in figure B1.4.5 [36]. The resulting resolution is truly exceptional and leads to among the most precise molecular constants ever determined. Pioneered in the former Soviet Union, $\mathrm{THz}$ BWOs are finding increased applications in a number of laboratories.

BWOs are typically placed in highly overmoded waveguides, and the extension of this technology to higher frequencies will, by necessity, require a number of innovative solutions to the very small-scale structures that must be fabricated. Their size and weight also preclude them from space-based applications (e.g. FIRST). Thus, electronic oscillators are unlikely to cover all THz spectroscopy and/or remote sensing applications over the full range of $1-10 \mathrm{THz}$ in the short term, and a number of alternative $\mathrm{THz}$ radiation sources are therefore under investigation. Among the most promising of these, over the long term, are engineered materials such as 


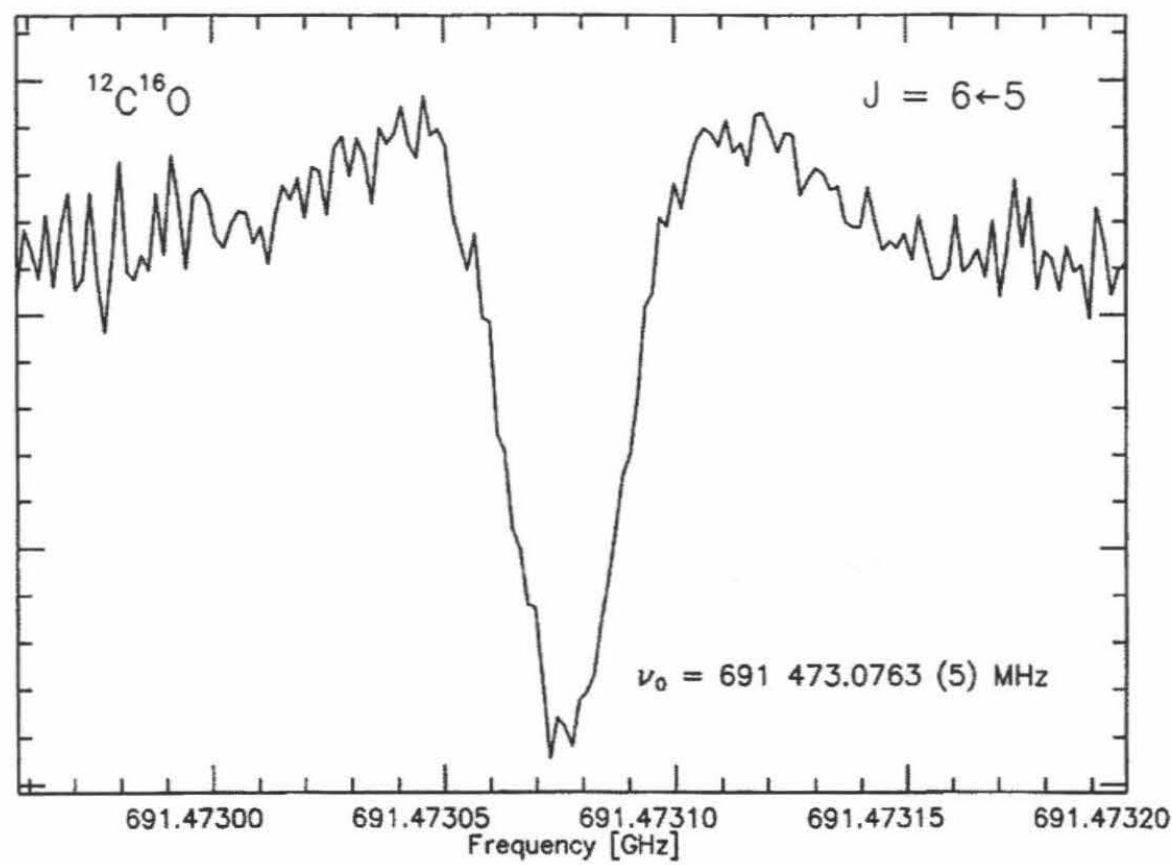

Figure B1.4.5. The Lamb dip spectrum of the $\mathrm{CO} 6-5$ transition obtained with the Cologne $\mathrm{THz} \mathrm{BWO}$ spectrometer. The dip is of order $30-40 \mathrm{kHz}$ in width and the transition frequency is determined to $0.5 \mathrm{kHz}$ [36].

quantum wells that either possess high nonlinearity for $\mathrm{THz}$ mixing experiments [37] or that can be tailored to provide direct emission in the FIR [38]. Tunable laser sources are the ultimate goal of such development programmes, but while $\mathrm{THz}$ spontaneous emission has been observed, laser action is still some time in the future.

A number of mixing experiments have therefore been used to generate both pulses and $\mathrm{CW}$ THz radiation. Among these, diode-based mixers used as upconvertors (that is, heterodyne spectroscopy 'in reverse') have been the workhorse FIR instruments. Two such techniques have produced the bulk of the spectroscopic results: (1) GaAs-based diode mixers that generate tunable sidebands on line-tunable FIR molecular gas lasers $[39,40]$, and (2) $\mathrm{CO}_{2}$ laser-based $\mathrm{THz}$ difference frequency generation in ultrafast metal-insulatormetal (MIM) diodes [41,42]. Both types of mixers have sufficient instantaneous bandwidth to place any desired millimetre-wave frequency on the carrier radiation, and respond well at $\mathrm{THz}$ frequencies. Having been used for many years in astronomical receiver applications, GaAs mixer technology is more mature than that for MIM diodes, and its conversion, noise and coupling mechanisms are better understood at present. In addition, their conversion efficiencies are good up to at least 4-5 THz, and several to several tens of microwatts are available from GaAs Schottky diode laser sideband generators. It is thus possible to construct $\mathrm{THz}$ spectrometers based on laser sideband generation that operate at or near the shot-noise limit, and this sensitivity has been used to investigate a wide range of interesting reactive and/or transient species, as is described in section B1.4.4.6.

While the conversion efficiency of MIM diodes is not as good as that of their GaAs counterparts, they are considerably faster, having also been used at IR and even visible wavelengths! Thus, MIM-based THz spectrometers work over wider frequency ranges than do GaAs FIR laser sideband generators, but with less output power. As described above, this translates directly into spectrometer sensitivity due to the high 
laboratory background in the THz region. Thus, where intense FIR gas laser lines are available, sideband generators are to be preferred, but at present only MIM-diode spectrometers can access the spectroscopically important region above $200 \mathrm{~cm}^{-1}$ [42]. Also, because it is easy to block $\mathrm{CO}_{2}$ laser radiation with a variety of reststrahlen solid-state filters, there is no fixed-frequency FIR gas laser carrier to reject in MIM spectrometers, and this simplifies the overall experimental design. MIM spectrometers that perform third-order mixing of two $\mathrm{CO}_{2}$ laser lines and a tunable microwave source have also been constructed. This approach leads to very wide tunability and eliminates the need to scan the $\mathrm{CO}_{2}$ lasers, and only decreases the output power by a small amount. Thus, it is possible to phase lock the $\mathrm{CO}_{2}$ and microwave sources, leading to a direct synthesis approach to $\mathrm{THz}$ radiation to very high frequencies indeed. This is not feasible for the FIR laser sideband generators, and so the MIM approach has provided extremely accurate $\mathrm{THz}$ frequency standards for calibration gases such as $\mathrm{CO}, \mathrm{HF}$ and $\mathrm{HCl}$, which can then be used as secondary standards in a number of other techniques [41].

While extremely useful in the laboratory, the size and power requirements of both FIR laser sideband generators and MIM-based $\mathrm{CO}_{2}$ laser spectrometers are excessive for space-borne applications. Research on other $\mathrm{THz}$ generation approaches by mixing has therefore continued. One particularly interesting approach from a technology and miniaturization point of view is optical heterodyne conversion, in which optical radiation is converted to $\mathrm{THz}$ light by semiconducting materials pumped above their band gaps. The use of optical or near-IR lasers to drive the process results in wide tunability and spectral coverage of the $\mathrm{THz}$ spectrum. Such approaches, described next, also have the considerable advantage of levering the rapid technological innovations in diode-pumped lasers and fast optoelectronic devices required for emerging industries such as optical telecommunications and optical computing. They therefore 'break the mould' of traditional THz LO development by small groups focused on scientific problems, and rapid developments can be expected with little or no investment by the $\mathrm{THz}$ community. Finally, as described next, both ultrafast time-resolved and $\mathrm{CW}$ high-resolution spectroscopies can be carried out using these approaches.

\section{B1.4.4.4 Time domain $\mathrm{THz}$ spectroscopy}

Free-electron lasers have long enabled the generation of extremely intense, sub-picosecond $\mathrm{THz}$ pulses that have been used to characterize a wide variety of materials and ultrafast processes [43]. Due to their massive size and great expense, however, only a few research groups have been able to operate them. Other approaches to the generation of sub-picosecond $\mathrm{THz}$ pulses have therefore been sought, and one of the earliest and most successful involved semiconducting materials. In a photoconductive semiconductor, carriers (for n-type material, electrons) in the valence band absorb the incident radiative power (if $h v_{0} \geq E_{\text {bandgap }}$ ) and are injected into the conduction band. Once they are in the conduction band, the electrons become mobile, and, if there is an applied bias, they begin to drift toward the photoconductor electrodes. Some of the electrons will reach the electrodes while some will encounter sites of ionic impurities. The latter electrons are trapped by the impurity sites and removed from the conduction band. As early as two decades ago, pulsed optical lasers were used by Auston and co-workers to generate and detect electrical pulses in DC biased voltage transmission lines [44]. For the earliest used materials such as $\mathrm{GaAs}$ and $\mathrm{Si}$, the pulse widths were of order nanoseconds due to their long recombination times. The discovery of materials such as radiation-damaged silicon-on-sapphire and of low-temperature-grown (LTG) GaAs changed this situation dramatically.

Especially with LTG GaAs, materials became available that were nearly ideal for time-resolved $\mathrm{THz}$ spectroscopy. Due to the low growth temperature and the slight As excess incorporated, clusters are formed which act as recombination sites for the excited carriers, leading to lifetimes of $\leq 250 \mathrm{fs}$ [45]. With such recombination lifetimes, THz radiators such as dipole antennae or log-periodic spirals placed onto optoelectronic substrates and pumped with ultrafast lasers can be used to generate sub-picosecond pulses with optical bandwidths of 2-4 THz. Moreover, coherent sub-picosecond detection is possible, which enables both the real and imaginary refractive indices of materials to be measured. The overall sensitivity is $>10^{4}$, and a 
variety of solid-state and gas phase $\mathrm{THz}$ spectra have been acquired with such systems [46, 47], an excellent overview of which may be found in [48].

Recently, it has been shown that both the detection and generation of ultrafast $\mathrm{THz}$ pulses can be carried out using the electro-optic effect in thin films of materials such as $\mathrm{ZnTe}$, GaAs and InP that are pumped in the near-IR [49]. The generation efficiency is similar to that of the photoconducting antenna approach, but the electro-optic scheme offers two extremely significant advantages. First, the detection bandwidth can be extremely large, up to $30-40 \mathrm{THz}$ under optimum conditions [49]. Second, it is possible to directly image the $\mathrm{THz}$ field with such spectrometers. Such approaches therefore make possible the $\mathrm{THz}$ imaging of optically opaque materials with a compact, all solid-state, room-temperature system [50]!

The great sensitivity and bandwidth of electro-optic approaches to optical-THz conversion also enable a variety of new experiments in condensed matter physics and chemistry to be conducted, as is outlined in figure B1.4.6. The left-hand side of this figure outlines the experimental approach used to generate ultrafast optical and $\mathrm{THz}$ pulses with variable time delays between them [51]. A mode-locked Ti:sapphire laser is amplified to provide approximately $1 \mathrm{~W}$ of $100 \mathrm{fs}$ near-IR pulses at a repetition rate of $1 \mathrm{kHz}$. The $\sim 850 \mathrm{~nm}$ light is divided into three beams, two of which are used to generate and detect the $\mathrm{THz}$ pulses, and the third of which is used to optically excite the sample with a suitable temporal delay. The right-hand panel presents the measured relaxation of an optically excited TBNC molecule in liquid toluene. In such molecules, the charge distribution changes markedly in the ground and electronically excited states. In TBNC, for example, the excess negative charge on the central porphyrin ring becomes more delocalized in the excited state. The altered charge distribution must be accommodated by changes in the surrounding solvent. This so-called solvent reorganization could only be indirectly probed by Stokes shifts in previous optical-optical pump-probe experiments, but the optical-THz approach enables the solvent response to be directly investigated. In this case, at least three distinct temporal response patterns of the toluene solvent can be seen that span several temporal decades [51]. For solid-state spectroscopy, ultrafast $\mathrm{THz}$ studies have enabled the investigation of coherent oscillation dynamics in the collective (phonon) modes of a wide variety of materials for the first time [49].

\section{B1.4.4.5 THz optical-heterodyne conversion in photoconductors}

For CW applications of optical-heterodyne conversion, two laser fields are applied to the optoelectronic material. The non-linear nature of the electro-optic effect strongly suppresses continuous emission relative to ultrashort pulse excitation, and so most of the $\mathrm{CW}$ research carried out to date has used photoconductive antennae. The CW mixing process is characterized by the average drift velocity $\bar{v}$ and carrier lifetime $\tau_{0}$ of the mixing material, typically LTG GaAs. If $\tau_{0} \bar{v} \geq \delta$, the electrode spacing, then a significant amount of current will be generated by the photo-excitation. That is

$$
i(t)=\frac{N_{\mathrm{c}}(t) e \bar{v}}{\delta}
$$

where $N_{\mathrm{c}}$ is the number of carriers. The rate equation for the photo-excitation-recombination process can be written as

$$
\frac{\mathrm{d} N_{\mathrm{c}}(t)}{\mathrm{d} t}=a P_{0}-\frac{N_{\mathrm{c}}(t)}{\tau_{0}}
$$

where $a$ is a proportionality constant and $P_{0}$ is the average power of the incident field over a few optical periods. The time-average is of interest here because $\tau_{0}$ in a semiconductor is always longer than an optical cycle and, therefore, the output current will not respond directly to the optical oscillations. Since the $\mathrm{THz}$ waves are generated with optical light, $\omega \equiv\left(\omega_{1}-\omega_{2}\right) \ll \omega_{1} \approx \omega_{2}$. Thus, integrating the right-hand side of the expression above over a few cycles of $\omega_{1} \approx \omega_{2}$ yields

$$
P_{0}=E_{1}^{2}+E_{2}^{2}+2 E_{1} E_{2} \cos \left(\omega t+\phi_{1}-\phi_{2}\right) .
$$


Substitution yields

$$
\frac{\mathrm{d} N_{\mathrm{c}}}{\mathrm{d} t}=a\left[E_{1}^{2}+E_{2}^{2}+2 E_{1} E_{2} \cos \left(\omega t+\phi_{1}-\phi_{2}\right)\right]-\frac{N_{\mathrm{c}}}{\tau_{0}} .
$$

By solving the differential equation above and using the single-pump case $\left(P_{2}=0\right)$ to determine $a$, it can be shown that the photo-current is

$$
i(t)=\frac{e \eta}{h \nu_{0}} \frac{\tau_{0} \bar{v}}{\delta}\left[P_{1}+P_{2}+2 \sqrt{\frac{P_{1} P_{2}}{1+\omega^{2} \tau_{0}^{2}}} \cos (\omega t-\phi)\right]
$$

where $v_{0} \equiv v_{1} \approx v_{2}, P_{1}$ and $P_{2}$ are the incident optical powers, $\phi=\tan ^{-1}\left(\omega \tau_{0}\right)$ and $\eta$, the quantum efficiency, is the number of carriers excited per incident photon. Recognizing that $\bar{v}=\mu E$ (where $\mu$ is the carrier mobility and $E$ is the electric field),

$$
i(t)=\frac{e \eta}{h v_{0}} \frac{\tau_{0} \mu E}{\delta}\left[P_{1}+P_{2}+2 \sqrt{\frac{P_{1} P_{2}}{1+\omega^{2} \tau_{0}^{2}}} \cos (\omega t-\phi)\right] .
$$

Separating the DC and oscillatory parts of the above equation gives

$$
\begin{gathered}
i_{\mathrm{dc}}=\frac{e \eta}{h v_{0}} \frac{\tau_{0} \mu E}{\delta}\left(P_{1}+P_{2}\right) \\
i_{\mathrm{THz}}(t)=\frac{e \eta}{h v_{0}} \frac{\tau_{0} \mu E}{\delta} 2 \sqrt{\frac{P_{1} P_{2}}{1+\omega^{2} \tau_{0}^{2}}} \cos (\omega t-\phi) .
\end{gathered}
$$

Thus, the beating of the two incident optical fields generates a modulation in the photo-excitation of the carriers, which in turn results in an oscillating electrical signal. Initial microwave experiments using an interdigitated electrode geometry by Brown et al [52] showed a flat frequency response up to $25 \mathrm{GHz}$ with a conversion efficiency of $0.14 \%$, in agreement with the signal level predicted by the theoretical analysis outlined above. At $\mathrm{THz}$ frequencies, the power decays rapidly from such structures due to the parasitic capacitance of the electrode structure and the finite carrier lifetime. Free-space radiation is generated by coupling the electrodes to a planar THz antenna. At $1 \mathrm{THz}$, the observed conversion efficiency is roughly $3 \times 10^{-6}$, and the damage threshold is of order $1 \mathrm{~mW} \mu \mathrm{m}^{-2}$. To alleviate these limitations, travelling-wave structures have now been developed that eliminate the capacitive roll-off and allow large-device active areas to be pumped. Powers in excess of $1 \mu \mathrm{W}$ can now be achieved above $2 \mathrm{THz}$ for input drive levels of 300-400 mW [53].

This power level is sufficient for laboratory spectroscopy or for use as a THz local oscillator, and such travelling-wave structures can be used over at least a decade of frequency $(0.3-3 \mathrm{THz}$, for example) without moving parts. Further, compact, all solid-state spectrometers, such as that outlined in figure B1.4.7, can now be constructed using CW diode laser and optical tapered amplifier technology [54]. The major challenge in working with diode lasers is, in fact, their instantaneous line widths $(\geq 15 \mathrm{MHz})$ and long-term frequency stability ( $100-200 \mathrm{MHz}$ ), both of which need considerable improvement to be useful as THz LOs. The main source of both instabilities is the notorious susceptibility of diode lasers to optical feedback. However, this susceptibility can be used to one's advantage by sending a small fraction of the laser output into a high-finesse $(F \geq 60)$ optical cavity such that the diode laser 'sees' optical feedback only at cavity resonances. By locking the diode lasers to different longitudinal modes of an ultrastable reference cavity, it is possible to construct direct synthesis spectrometers that can be absolutely calibrated to $\Delta v / v<10^{-8}$ or better. To demonstrate the continuous tunability and frequency stability of such an instrument, the lower panel of figure B1.4.7 presents a submillimetre spectrum of acetonitrile in which the transition frequencies are measured to better than $50 \mathrm{kHz}$. Future improvements to such systems should allow similar measurements on both stable and transient species up to at least 5-6 THz. 


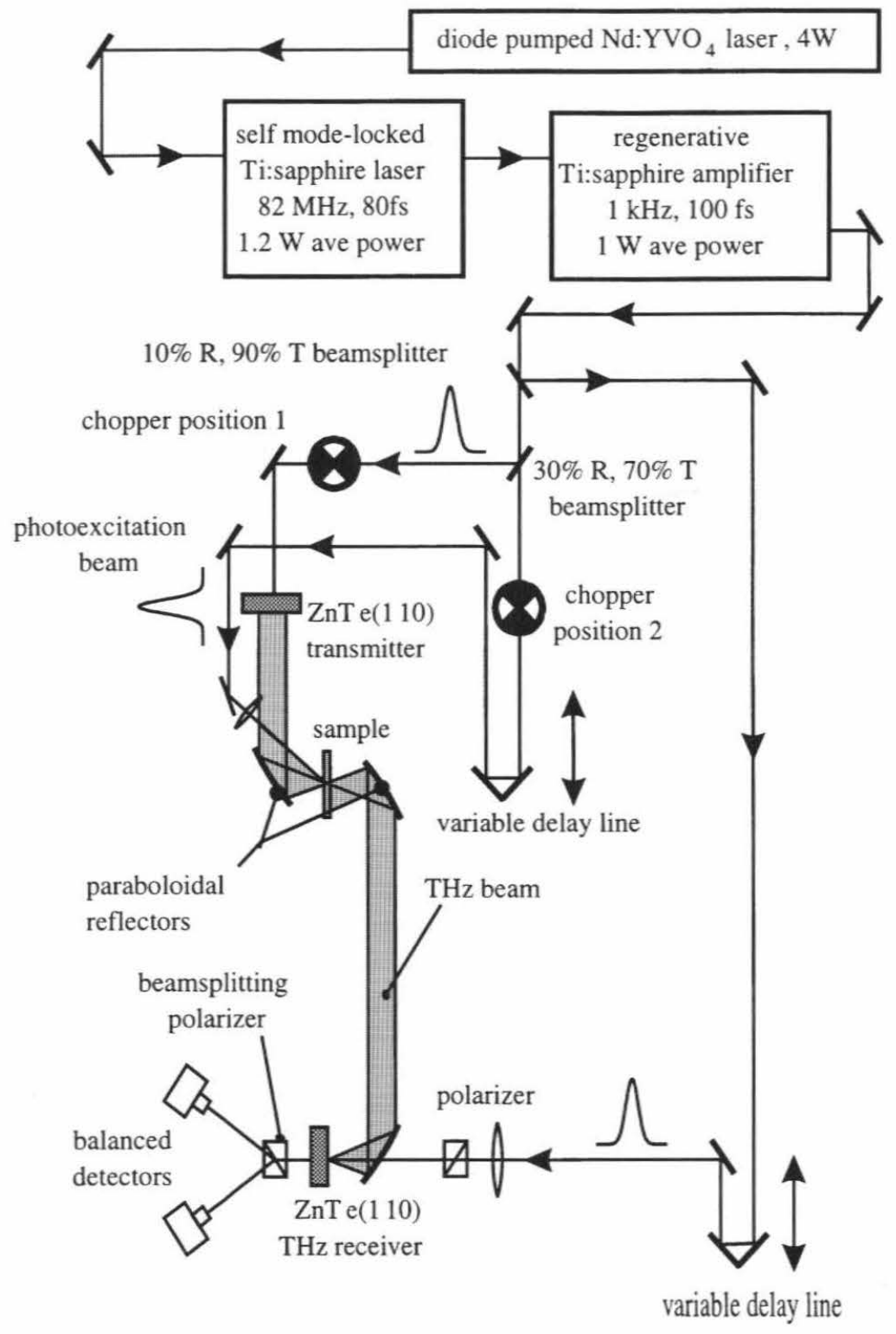

(a)

Figure B1.4.6. Left: an experimental optical $\mathrm{THz}$ pump-probe set-up using sub-picosecond $\mathrm{THz}$ pulse generation and detection by the electro-optic effect. Right: the application of such pulses to the relaxation of optically excited TBNC in toluene. The $\mathrm{THz}$ electric field used for these experiments is shown in the upper-right inset. Three exponential decay terms, of order 2, 50 and $700 \mathrm{ps,}$, are required to fit the observed temporal relaxation of the solvent [51].

\section{B1.4.4.6 THz spectroscopy of hydrogen-bonded and refractory clusters}

Among the most interesting transient species that can be studied at $\mathrm{THz}$ frequencies are those involving collections of molecules held together by van der Waals or hydrogen bonding forces. In no small measure this is true 


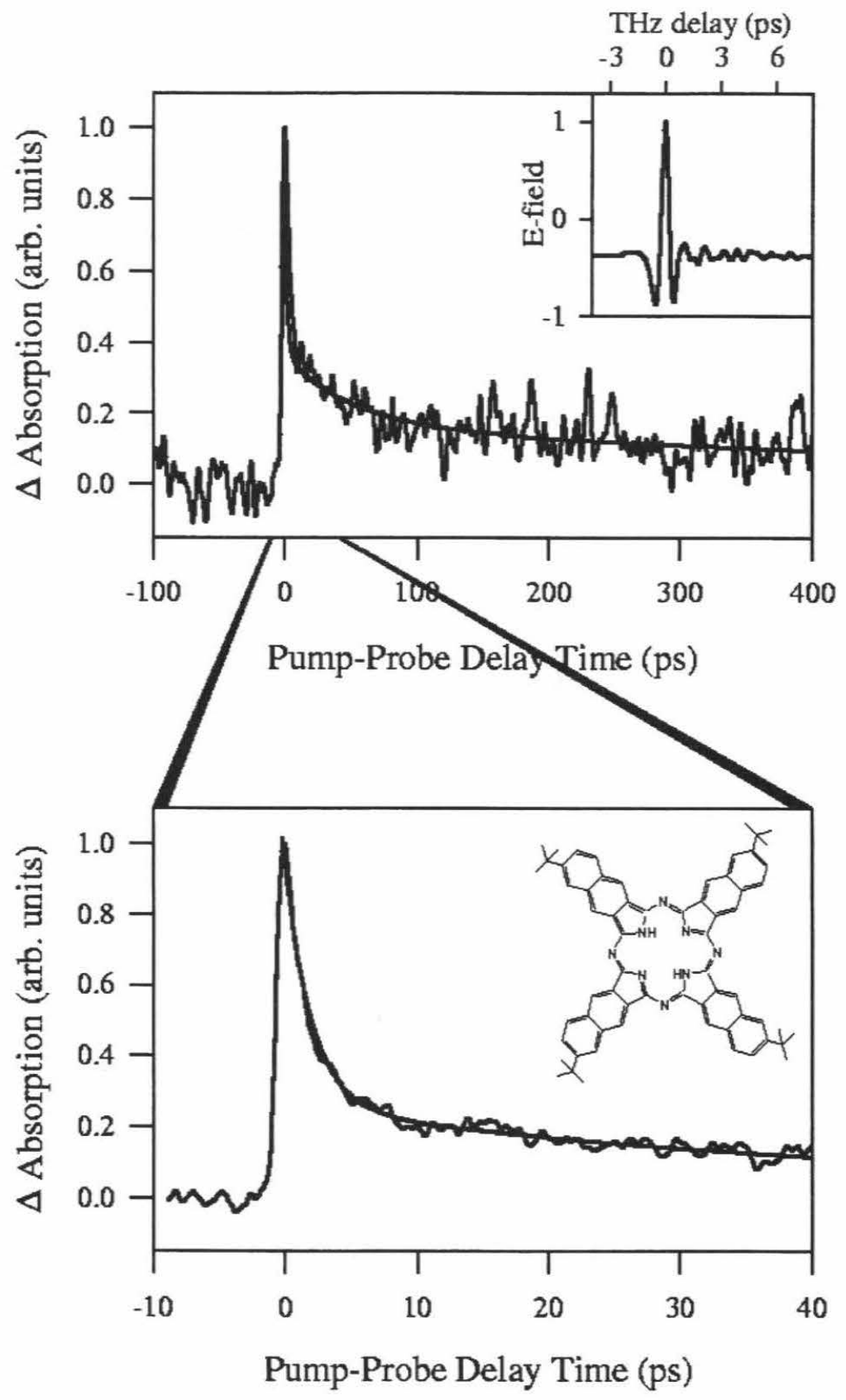

(b)

Figure B1.4.6. (Continued)

because hydrogen bonds are ubiquitous in nature. From the icy mantles covering interstellar dust to the nuclei of living cells, hydrogen bonds play crucial roles in the regulation and evolution of both inorganic and living systems. Accurate, fully anisotropic, descriptions of the intermolecular forces involved in these and other weak 
(a)

(b)
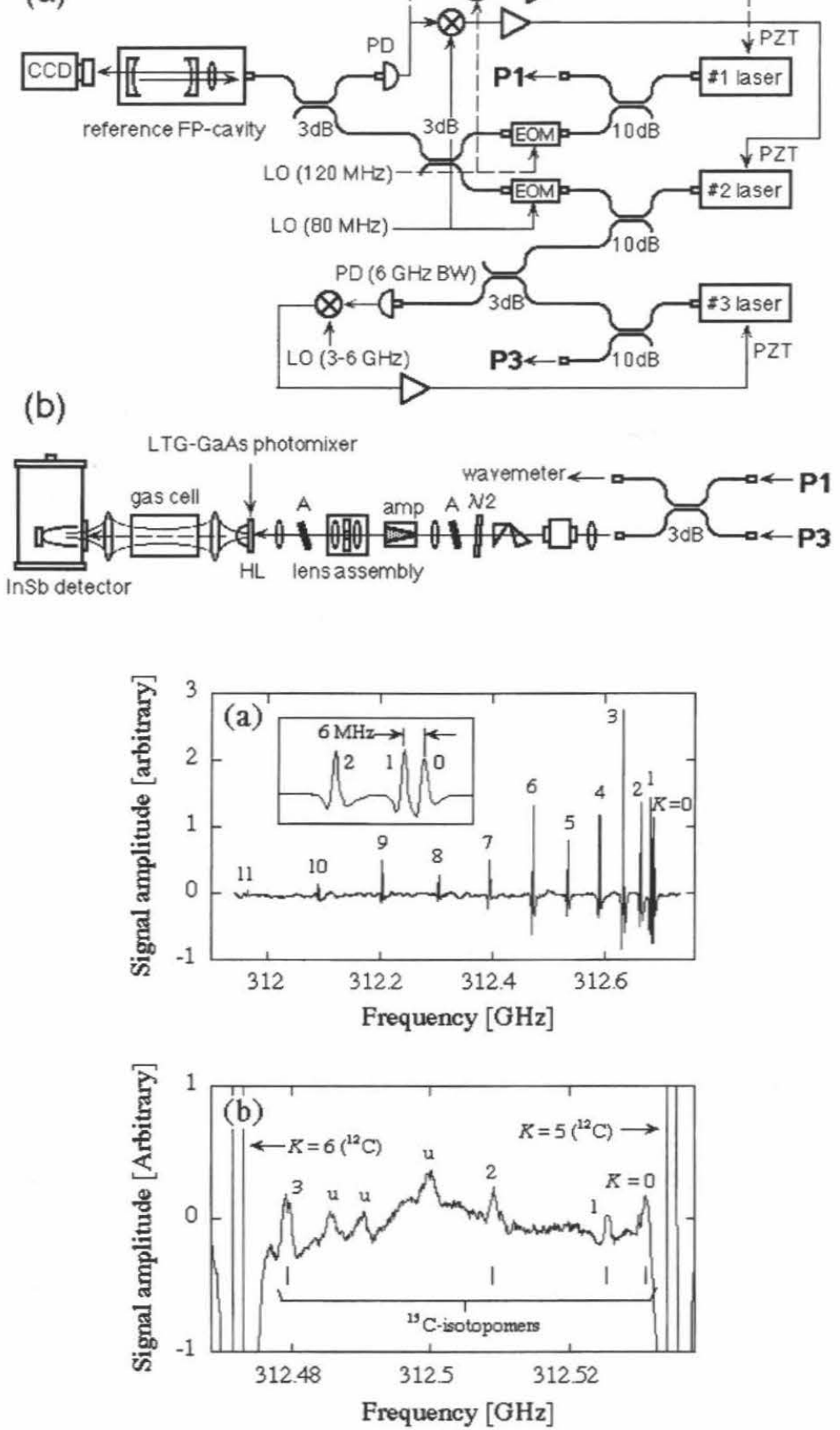

Figure B1.4.7. Top: THz generation by optical-heterodyne conversion in low-temperature GaAs. (a) The three DBR laser system that synthesizes a precise difference frequency for the $\mathrm{THz}$ photomixer spectrometer, (b) the MOPA system and the set-up for spectroscopy. Bottom: second-derivative absorption spectrum of the $\mathrm{CH}_{3} \mathrm{CN} \mathrm{J}_{K}=16_{K} \rightarrow 17_{K}$ rotational transitions near $312 \mathrm{GHz}$. (a) The spectrum for ordinary ${ }^{12} \mathrm{CH}_{3}{ }^{12} \mathrm{CN}$. The inset is an expanded view of the $K=0-2$ lines. (b) The $K=0-3$ lines of $\mathrm{CH}_{3}{ }^{13} \mathrm{CN}$ [54]. 
interactions are therefore assuming an increasingly pivotal role in modern molecular science, particularly in molecular biology [54]. Within chemical physics, the anisotropy of intermolecular forces plays a central role in understanding the dynamics associated with photoinitiated reactions in clusters [55], to name but one example.

Over the past century, much of the data underlying current descriptions of van der Waals and hydrogen bonds were obtained from measurements of second virial coefficients, pressure broadening, and other classical properties. Experimental advances during the past three decades have led to many techniques capable of interrogating intermolecular forces, most notably scattering experiments and the spectroscopic study of isolated clusters. Despite this long-standing interest, however, truly quantitative, microscopic models of these forces have only become available in recent years as advances in ab initio theory, high-resolution spectroscopy and eigenvalue generation on complex potential energy surfaces have converged, to enable the fitting of fully anisotropic force fields to experimental data for systems with two, three and four degrees of freedom [57].

The weak interactions in clusters are mathematically modelled by means of a multi-dimensional intermolecular potential energy surface, or IPS. Microwave spectroscopy, carried out primarily with the elegant, extraordinarily sensitive and sub-Doppler resolution FTMW technique outlined previously, proves the very lowest region of the ground-state surface; visible and IR spectroscopy probes states above the dissociation energy of the adduct (the latter of which are described elsewhere in this encyclopaedia). None are, in themselves, direct probes of the total ground-state IPS. Indeed, while microwave, IR and UV/Vis instruments have produced structural parameters and dynamical lifetimes for literally dozens of binary (and larger) weakly bound complexes (WBCs) over the past two decades [58-61], recent calculations which explicitly allow coupling between all the degrees of freedom present in the cluster reveal that structural parameters alone are not sufficient to accurately characterize the IPS [62].

Weak interactions are characterized by binding energies of at most a few kcal/mole and by IPSs with a very rich and complex topology connected by barriers of at most a few hundred $\mathrm{cm}^{-1}$. Rotational, tunnelling and intermolecular vibrational states can therefore become quite strongly mixed, hence the general term of vibration-rotation-tunnelling (VRT) spectroscopy for the study of eigenvalues supported by an IPS [63, 64]. The VRT states in nearly all systems lie close to or above the tunnelling barriers, and therefore sample large regions of the potential surface. In addition, as they become spectroscopically observable, the number, spacings and intensities of the tunnelling splittings are intimately related to the nature of the tunnelling paths over the potential surface.

Thus, by measuring the intermolecular vibrations of a WBC, ultimately with resolution of the rotational, tunnelling and hyperfine structure, the most sensitive measure of the IPS is accessed directly. The difficulty of measuring these VRT spectra is the fact that they lie nearly exclusively at THz frequencies. As expected, the 'stiffer' the interaction, the higher in frequency these modes are found. In general, the total $0.3-30 \mathrm{THz}$ interval must be accessed, although for the softest or heaviest species the modes rarely lie above 10-15 THz.

For WBCs composed of stable molecules, planar jet expansions produce sufficiently high concentrations that direct absorption $\mathrm{THz}$ studies can be pursued for clusters containing $\lesssim 6$ small molecules using FIR laser sidebands. Research on water clusters has been particularly productive, and has been used to investigate the structures and large-amplitude dynamics of the clusters outlined in the top panel of figure B1.4.8 [65-68]. In addition, as the bottom panel illustrates, not only are the VRT modes directly sampled by such work, but the available spectral resolution of $\lesssim 1 \mathrm{MHz}$ enables full rotational resolution along with a detailed investigation of the VRT and hyperfine splittings. The high resolution is also essential in untangling the often overlapping bands from the many different clusters formed in the supersonic expansion.

For clusters beyond the dimer, each of the monomers can both accept and donate hydrogen bonds, which leads to a rich suite of large-amplitude motions. Their spectroscopic manifestations are illustrated for the water trimer in figure B1.4.9. The most facile motion in this system is the 'flipping' of one of the non-bonded hydrogen atoms through the plane of the oxygen atoms. This motion is sufficiently fast that it produces symmetric top rovibrational spectra even though at any one instant the molecule is always asymmetric. Six of these flipping motions lead to the same structure, a process know as 'pseudorotation', and leads to the manifold 


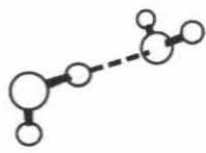

dimer

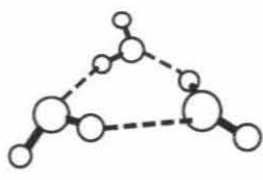

trimer

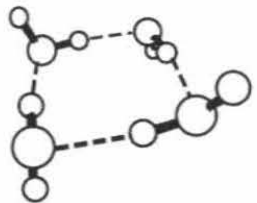

tetramer

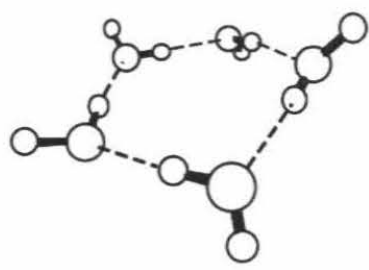

pentamer

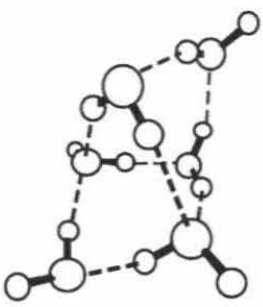

hexamer

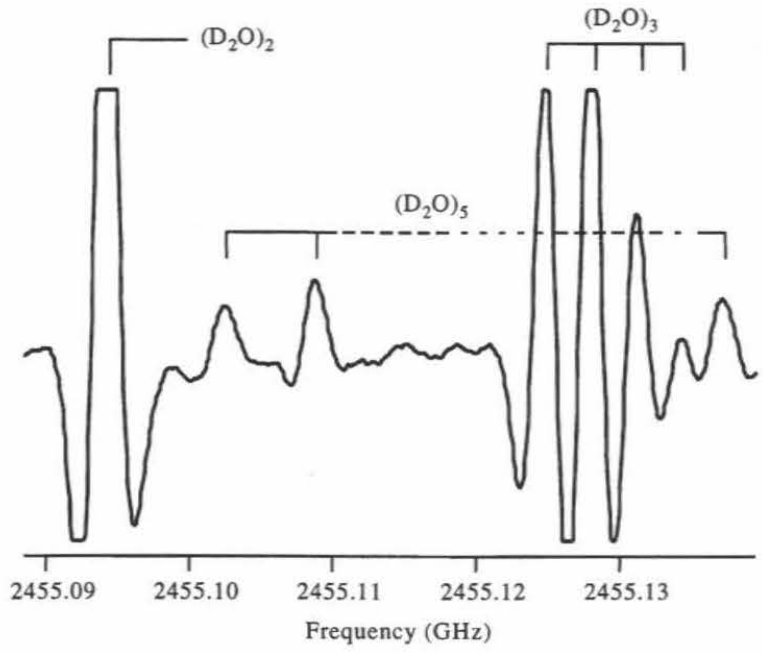

Figure B1.4.8. Top: the lowest-energy structures of water clusters, $\left(\mathrm{H}_{2} \mathrm{O}\right)_{n}$, from $n=2-6$. Bottom: a sample $\sim 2.5 \mathrm{THz}$ spectrum of such clusters formed in a pulsed planar supersonic expansion [65].

of states produced in the bottom panel of figure B1.4.9. The exchange of bound versus free hydrogen atoms in a monomer leads to the hyperfine splittings of the individual transitions, as is illustrated in the top panel of the same figure. From a comparison of such spectra with detailed calculations, a variety of IPS properties can be extracted to experimental precision.

Molecules like those presented in figure B1.4.4 form another interesting suite of targets from a $\mathrm{THz}$ perspective. Such chains can be treated as rigid rods, and as they get longer their lowest bending frequencies 

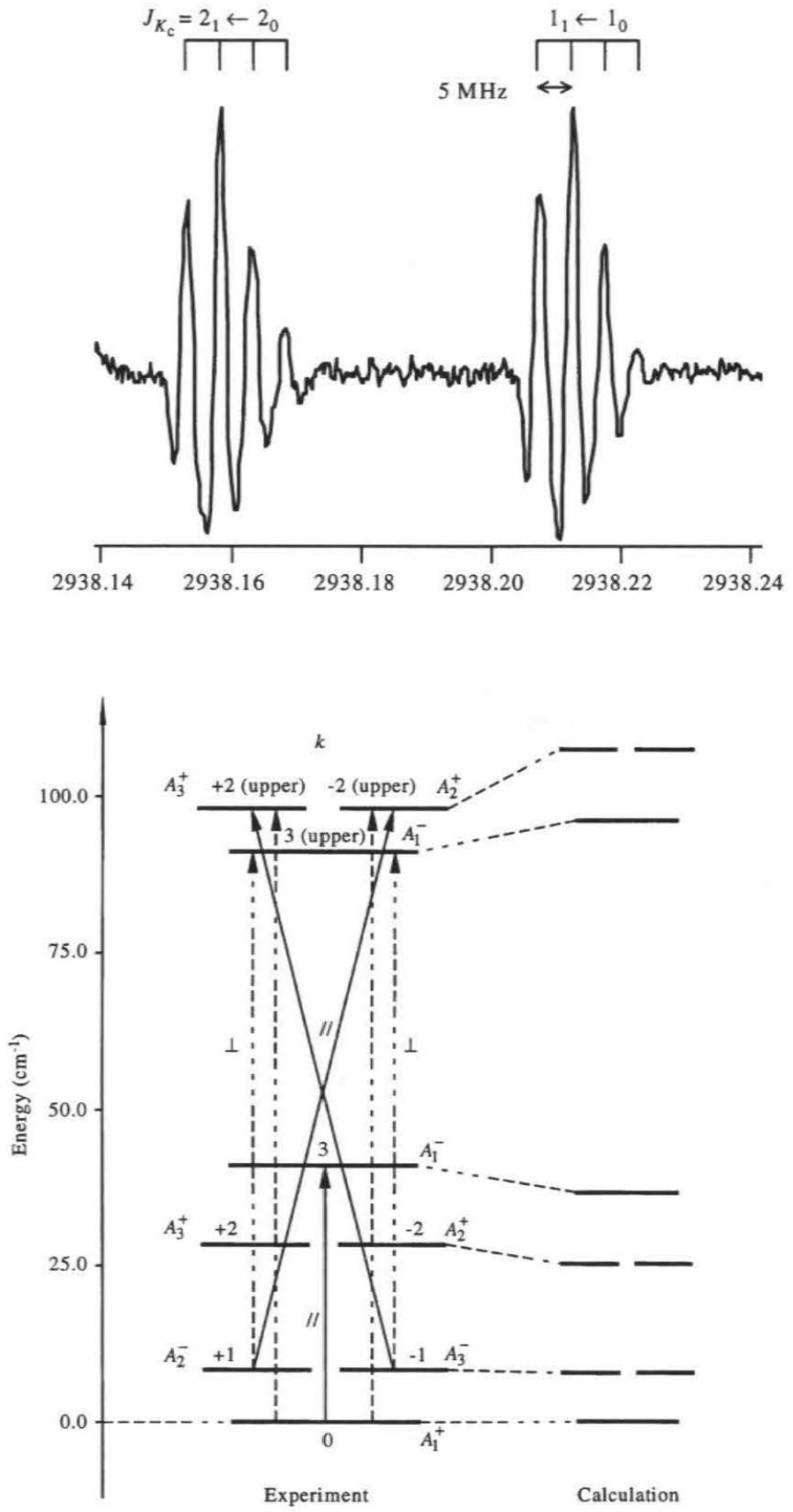

Figure B1.4.9. Top: rotation-tunnelling hyperfine structure in one of the 'flipping' modes of $\left(\mathrm{D}_{2} \mathrm{O}\right)_{3}$ near $3 \mathrm{THz}$. The small splittings seen in the $Q$-branch transitions are induced by the bound-free hydrogen atom tunnelling by the water monomers. Bottom: the low-frequency torsional mode structure of the water dimer spectrum, including a detailed comparison of theoretical calculations of the dynamics with those observed experimentally [65]. The symbols next to the arrows depict the parallel $(\Delta k=0)$ versus perpendicular $(\Delta K= \pm 1)$ nature of the selection rules in the pseudorotation manifold. 
move rapidly into the FIR. For example, the lowest frequencies of a variety of chains are as follows:

$$
\begin{array}{lrl}
\text { Cyanopolyynes, } \mathrm{HC}_{3} \mathrm{~N} \rightarrow \mathrm{HC}_{25} \mathrm{~N} & 222 \rightarrow 8 \mathrm{~cm}^{-1} \\
\text { Polyacetylenes, } \mathrm{HC}_{4} \mathrm{~N} \rightarrow \mathrm{HC}_{20} \mathrm{H} & 220 \rightarrow 19 \mathrm{~cm}^{-1} \\
\text { Carbon clusters, } \mathrm{C}_{3} \rightarrow \mathrm{C}_{20} & 63 \rightarrow 17 \mathrm{~cm}^{-1} \\
\mathrm{C}_{n} \mathrm{~N} \text { radicals, } \mathrm{C}_{3} \mathrm{~N} \rightarrow \mathrm{C}_{19} \mathrm{~N} & 144 \rightarrow 4 \mathrm{~cm}^{-1} .
\end{array}
$$

A real advantage of working in the FIR is that both polar and non-polar chains may be searched for. Indeed, the lowest bending frequency of $\mathrm{C}_{3}$ has been studied in the laboratory [69], and tentatively detected toward the galactic centre source Sgr B2 [70]. Other large molecules such as polycyclic aromatic hydrocarbons (anthracene, pyrene, perylene, etc) or 'biomolecules' such as glycine or uracil also possess low-frequency FIR vibrations, and can be produced in sizable quantities in supersonic expansions through heated planar nozzles [71]. The study of such species is important cosmochemically, but is quite difficult at microwave frequencies where the rotational spectra are weak, and nearly impossible at IR or optical wavelengths due to the extinction present in dense molecular clouds and young stellar objects.

\section{B1.4.5 Outlook}

Technology developments are revolutionizing the spectroscopic capabilities at $\mathrm{THz}$ frequencies. While no one technique is ideal for all applications, both $\mathrm{CW}$ and pulsed spectrometers operating at or near the fundamental limits imposed by quantum mechanics are now within reach. Compact, all-solid-state implementations will soon allow such spectrometers to move out of the laboratory and into a wealth of field and remote-sensing applications. From the study of the rotational motions of light molecules to the large-amplitude vibrations of clusters and the collective motions of condensed phases, microwave and $\mathrm{THz}$ spectroscopy opens up new windows to a wealth of scientifically and technologically important fields. Over the coming decade, truly user-friendly and extraordinarily capable instruments should become cost affordable and widely available, enabling this critical region of the electromagnetic spectrum to be fully exploited for the first time.

\section{References}

[1] Johns J W C 1985 High resolution far-infrared $\left(20-350 \mathrm{~cm}^{-1}\right)$ spectra of several isotopic species of $\mathrm{H}_{2} \mathrm{O} J$. Opt. Soc. Am. B 2 $1340-54$

[2] McLean I S 1995 Infrared array detectors-performance and prospects IAU Symp. 167 69-78

[3] Kimmitt M F 1970 Far-Infrared Techniques (London: Pion)

[4] Bock J J, Chen D, Mauskopf P D and Lange A E 1995 A novel bolometer for infrared and millimeter-wave astrophysics Space Sci. Rev. $74229-35$

[5] Lis D C, Serabyn E, Keene J, Dowell C D, Benford D J, Phillips T G, Hunter T R and Wang N 1998350 micro continuum imaging of the Orion A molecular cloud with the submillimeter high angular resolution camera (SHARC) Astrophys. J. 509 299-308

[6] Ivison R J, Smail I, Le Borgne J F, Blain A W, Kneib J P, Bezecourt J, Kerr T H and Davies J K 1997 A hyperluminous galaxy at $z=2.8$ found in a deep submillimetre survey Mon. Not. R. Astron. Soc. $298583-93$

[7] Putley E H 1964 The ultimate sensitivity of sub-mm detectors Infra. Phys. 4 1-35

[8] Beeman J W and Haller E E 1994 Ga:Ge photoconductor arrays-design considerations and quantitative analysis of prototype single pixels Infra. Phys. Technology $35827-36$

[9] Waters J W 1993 Microwave limb sounding Atmospheric Remote Sensing by Microwave Radiometry ed M A Janssen (New York: Wiley) pp 383-496

[10] Chance K, Traub W A, Johnson D G, Jucks K W, Ciarpallini P, Stachnik R A, Salawitch R J and Michelsen H A 1996 Simultaneous measurements of stratospheric $\mathrm{HO}_{x}, \mathrm{NO}_{x}$, and $\mathrm{Cl}_{x}$ : comparison with a photochemical model J. Geophys. Res. $1019031-43$

[11] Winnewisser G 1994 Submillimeter and infrared astronomy: recent scientific and technical developments Infra. Phys, Tech. 35 $551-67$

[12] Carlstron J and Zmuidzinas J 1996 Millimeter and submillimeter techniques Review of Radio Science 1993-1996 ed W Ross Stone (Oxford: Oxford University Press) pp 839-92

[13] Bin M, Gaidis M C, Zmuidzinas J and Phillips T G 1997 Quasi-optical SIS mixers with normal tuning structures IEEE Trans. Appl. Supercond. $73584-8$ 
[14] Karasik B S, Gaidis M C, McGrath W R, Bumble B and LeDuc H G 1997 A low noise 2.5 THz superconducting NB hot-electron mixer IEEE Trans. Appl. Supercond. 7 3580-3

[15] Barrett A H 1983 The beginnings of molecular radio astronomy Serendipitous Discoveries in Radio Astronomy ed K Kellerman and B Sheets (Green Bank, WV: NRAO)

[16] Ohishi M 1997 Observations of hot cores IAU Symp. 178 61-74

[17] Phillips T G and Keene J 1992 Submillimeter astronomy Proc. IEEE 80 1662-78

[18] Hartquist T W and Williams D A (eds) 1998 The Molecular Astrophysics of Stars and Galaxies (Oxford: Oxford University Press)

[19] Herbst E 1995 Chemistry in the interstellar medium Ann. Rev. Phys. Chem. 46 27-53

[20] van Dishoeck E F and Blake G A 1998 Chemical evolution of star forming regions Ann. Rev. Astron. Ap. 36 $317-68$

[21] van Dishoeck E F 1997 The importance of high resolution far-infrared spectroscopy of the interstellar medium Proc. ESA Symp. SP-401 81-90

[22] Dutrey A, Guilloteau S and Guelin M 1997 Chemistry of protosolar-like nebulae: the molecular content of the DM Tau and GG Tau disks Astron. Astrophys. 317 L55-8

[23] Sargent A I 1997 Protostellar and protoplanetary disks IAU Symp. 170 151-8

[24] Biver N et al 1997 Evolution of the outgassing of comet Hale-Bopp (C/1995 O1) from radio observations Science 275 1915-18

[25] For an overview of ALMA, see http://www.nrao.edu

[26] Erickson E F 1995 SOFIA - the next generation airborne observatory Space Sci. Rev. 74 91-100

[27] Batchelor M, Adler D and Trogus W 1996 New plans for FIRST Missions to the Moon \& Exploring the Cold Universe 18 $185-8$

[28] Balle T J and Flygare W H 1981 A Fourier transform microwave spectrometer Rev. Sci. Instrum. 52 33-45

[29] Munrow M R, Pringle W C and Novick S E 1999 Determination of the structure of the argon cyclobutanone van der Waals complex J. Chem. Phys. $1032256-61$

[30] Lovas F J, Suenram R D, Ogata T and Yamamoto S 1992 Microwave spectra and electric dipole moments for low-J levels of interstellar radicals- $\mathrm{SO}, \mathrm{CCS}, \mathrm{CCCS}, \mathrm{c}-\mathrm{H}_{3}, \mathrm{CH}_{2} \mathrm{CC}$ and c- $\mathrm{C}_{3} \mathrm{H}_{2}$ Astrophys. J. 399 325-9

[31] Thaddeus P, McCarthy M C, Travers M, Gottlieb C and Chen W 1998 New carbon chains in the laboratory and in interstellar space Faraday Soc. Discuss. 109 121-36

[32] McCarthy M C, Travers M J, Kovacs A, Chen W, Novick S E, Gottlieb C A and Thaddeus P 1997 Science 275 $518-20$

[33] Gordy W 1960 Proc. Symp. MM Waves (Brooklyn: Polytechnic Press)

[34] Rothermel H, Phillips T G and Keene J 1989 Int. J. Infra. MM Waves 10 83-100

[35] Lewen F, Gendriesch R, Pak I, Paveliev D G, Hepp M, Schider R and Winnewisser G 1998 Phase locked backward wave oscillator pulsed beam spectrometer in the submillimeter wave range Rev. Sci. Instrum. 69 32-9

[36] Winnewisser G, Belov S P, Klaus T and Schieder R 1997 Sub-Doppler measurements on the rotational transitions of carbon monoxide J. Mol. Spectrosc. 184 468-72

[37] Maranowski K D, Gossard A C, Unterrainer K and Gornik E 1996 Far-infrared emission from parabolically graded quantum wells Appl. Phys. Lett. $693522-4$

[38] Xu B, Hu Q and Melloch M R 1997 Electrically pumped tunable terahertz emitter based on inter-subband transitions Appl. Phys. Lett. $71440-2$

[39] Bicanic D D, Zuiberg B F J and Dymanus 1978 Appl. Phys. Lett. 32 367-9

[40] Blake G A, Laughlin K B, Cohen R C, Busarow K L, Gwo D H, Schmuttenmaer C A, Steyert D W and Saykally R J 1991 Tunable far-infrared spectrometers Rev. Sci. Instrum. 62 1693-700

[41] Varberg T D and Evenson K M 1992 Accurate far-infrared rotational frequencies of carbon monoxide Astrophys. J. 385 763-5

[42] Odashima H, Zink L R and Evenson K M 1999 Tunable far-infrared spectroscopy extended to 9.1 THz Opt. Lett. 24 406-7

[43] Kono J, Su M Y, Inoshita T, Noda T, Sherwin M S, Allen S J and Sakaki H 1997 Resonant THz optical sideband generation from confined magnetoexcitons Phys. Rev. Lett. 79 1758-61

[44] For a historical review see Lee C H 1984 Picosecond Optoelectronic Devices (New York: Academic)

[45] Gupta S, Frankel M Y, Valdmanis J A, Whitaker J F, Mourou G A, Smith F W and Calawa A R 1991 Subpicosecond carrier lifetime in GaAs grown by MBE at low temperatures Appl. Phys. Lett. $593276-8$

[46] Jeon T I and Grischkowsky D 1998 Characterization of optically dense, doped semiconductors by reflection THz time domain spectroscopy Appl. Phys. Lett. 72 3032-4

[47] Cheville R A and Grioschkowsky D 1999 Far-infrared foreign and self-broadened rotational linewidths of high temperature water vapor J. Opt. Soc. Am. B 16 317-22

[48] Nuss M C and Orenstein J 1998 Terahertz time domain spectroscopy Millimeter Submillimeter Wave Spectrosc. Solids 74 7-50

[49] Han P Y, Cho G C and Zhang X C 1999 Mid-IR THz beam sensors: exploration and application for phonon spectroscopy, ultrafast phenomena in semiconductors III Proc. SPIE 3624 224-33

[50] Koch M, Hunsche S, Schuacher P, Nuss M C, Feldmann J and Fromm J 1998 THz-imaging: a new method for density mapping of wood Wood Sci. Technol. 32 421-7

[51] Venables D S and Schmuttenmaer C A 1998 Far-infrared spectra and associated dynamics in acetonitrile-water mixtures measured with femtosecond THz pulse spectroscopy J. Chem. Phys. 108 4935-44

[52] Brown E R, McIntosh K A, Smith F W, Manfra M J and Dennis C L 1993 Measurements of optical-heterodyne conversion in low-temperature grown GaAs Appl. Phys. Lett. 62 1206-8 
[53] Mastuura S, Blake G A, Wyss R, Pearson J, Kadow C, Jackson A and Gossard A C 1999 A travelling-wave photomixer based on angle-tuned phase matching Appl. Phys. Lett. 74 2872-4

[54] Matsuura S, Chen P, Blake G A, Pearson J and Pickett H M 1999 A tunable, cavity-locked diode laser system for terahertz photomixing IEEE Micro. Theory Technol. 48 380-7

[55] Stone A J 1996 The Theory of Intermolecular Forces (Oxford: Oxford University Press)

[56] Ionov S I, Ionov P I and Wittig C 1994 Time resolved studies of photoinitiated reactions in binary and larger $\left(\mathrm{N}_{2} \mathrm{O}\right)_{m}(\mathrm{HI})_{n}$ complexes Discuss. Faraday Soc. 97 391-400

[57] Cohen R C and Saykally R J 1991 Multidimensional intermolecular potential surfaces from VRT spectra of van der Waals complexes Ann. Rev. Phys. Chem. 42 369-92

[58] Novick S, Leopold K and Klemperer W 1990 Atomic and Molecular Clusters ed E Bernstein (New York: Elsevier) p 359-91 (the clusters listing presented here is now maintained electronically by $\mathrm{S}$ Novick)

[59] Miller R E 1988 Science 240 447-52

[60] Nesbitt D J 1990 Dynamics of Polyatomic van der Waals Complexes ed N Halberstadt and K C Janda (New York: Plenum) pp 461-70

[61] Chuang C, Andrews P M and Lester M I 1995 Intermolecular vibrations and spin-orbit predissociation dynamics of NeOH J. Chem. Phys. 103 3418-29

[62] Leforestier C, Braly L B, Liu K, Elrod M J and Saykally R J 1997 Fully coupled 6-dimensional calculations of the water dimer VRT states with a split Wigner pseudo-spectral approach J. Chem. Phys. 106 8527-44

[63] Saykally R J and Blake G A 1993 Molecular interactions and hydrogen bond tunneling dynamics: some new perspectives Science $2591570-5$

[64] Cotti G, Linnartz H, Meerts W L, van der Avoird A and Olthof E 1996 Stark effect and dipole moments of $\left(\mathrm{NH}_{3}\right)_{2}$ in different vibration-rotation-tunneling states J. Chem. Phys. 104 3898-906

[65] Liu K 1997 PhD Thesis University of California at Berkeley

[66] Viant M R, Cruzan J D, Lucas D D, Brown M G, Liu K and Saykally R J 1997 Pseudorotation in water trimer isotopomers using terahertz laser spectroscopy J. Phys. Chem. $1019032-41$

[67] Cruzan J D, Viant M R, Brown M G, Lucas D D, Liu K and Saykally R J 1998 Terahertz laser vibration-rotation-tunneling spectrum of the water pentamer-d(10). Constraints on the bifurcation tunneling dynamics Chem. Phys. Lett. 292 667-76

[68] Liu K, Brown M G and Saykally R J 1997 Terahertz laser vibration rotation tunneling spectroscopy and dipole moment of a cage form of the water hexamer J. Phys. Chem. $1018995-9010$

[69] Schmuttenmaer C A, Cohen R C, Pugliano N, Heath J R, Cooksy A L, Busarow K L and Saykally R J 1990 Tunable far-IR laser spectroscopy of jet-cooled carbon clusters-the $\nu_{2}$ bending vibration of $\mathrm{C}_{3}$ Science 249 897-900

[70] Heath J R, van Orden A, Hwang H J, Kuo E W, Tanaka K and Saykally R J 1994 Toward the detection of pure carbon clusters in the ISM Adv. Space Res. 15 25-33

[71] Liu K, Fellers R S, Viant M R, McLaughlin R P, Brown M G and Saykally R J 1996 A long pathlength pulsed slit valve appropriate for high temperature operation-infrared spectroscopy of jet-cooled large water clusters and nucleotide bases Rev. Sci. Instrum. $67410-16$

\section{Further Reading}

Kimmitt M F 1970 Far-Infrared Techniques (London: Pion)

An excellent overview of optical approaches to $\mathrm{THz}$ spectrometers.

Janssen M A (ed) 1993 Atmospheric Remote Sensing by Microwave Radiometry (New York: Wiley)

The most complete guide to microwave and $\mathrm{THz}$ atmospheric sensing.

Carlstrom J and Zmuidzinas J 1996 Millimeter and submillimeter techniques Review of Radio Science 1993-1996 ed W Ross Stone (Oxford: Oxford University Press) pp 839-82

A quite readable summary of heterodyne detection strategies.

Tsen K T (ed) 1999 Ultrafast phenomena in semiconductors III Proc. SPIE 624298

An overview of recent progress in this explosive field.

Gordy W and Cook R J 1991 Microwave Molecular Spectra (New York: Wiley)

The most complete textbook available, suitable for graduate students and researchers.

Stone A J 1996 The Theory of Intermolecular Forces (Oxford: Oxford University Press)

A thorough, advanced tutorial on the nature of clusters held together by intermolecular forces, and the theories that can be used to analyse them. 